\title{
A Review of Elliptic Flow of Light Nuclei in Heavy-Ion Collisions at RHIC and LHC Energies
}

\author{
Md. Rihan Haque, ${ }^{1}$ Chitrasen Jena, ${ }^{2,3}$ and Bedangadas Mohanty ${ }^{2}$ \\ ${ }^{1}$ Utrecht University, P.O. Box 80000, 3508 TA Utrecht, Netherlands \\ ${ }^{2}$ School of Physical Sciences, National Institute of Science Education and Research, Jatni 752050, India \\ ${ }^{3}$ Indian Institute of Science Education and Research, Tirupati 517507, India
}

Correspondence should be addressed to Md. Rihan Haque; r.haque@uu.nl

Received 30 June 2016; Revised 27 September 2016; Accepted 29 May 2017; Published 15 August 2017

Academic Editor: Shi-Hai Dong

Copyright (C) $2017 \mathrm{Md}$. Rihan Haque et al. This is an open access article distributed under the Creative Commons Attribution License, which permits unrestricted use, distribution, and reproduction in any medium, provided the original work is properly cited. The publication of this article was funded by SCOAP S $^{3}$

\begin{abstract}
We present a review of the measurements of elliptic flow $\left(v_{2}\right)$ of light nuclei $\left(d, \bar{d}, t,{ }^{3} \mathrm{He}\right.$, and $\left.{ }^{3} \overline{\mathrm{He}}\right)$ from the RHIC and LHC experiments. Light (anti)nuclei $v_{2}$ have been compared with that of (anti)proton. We observed a similar trend in light nuclei $v_{2}$ to that in identified hadron $v_{2}$ with respect to the general observations such as $p_{T}$ dependence, low $p_{T}$ mass ordering, and centrality dependence. We also compared the difference of nuclei and antinuclei $v_{2}$ with the corresponding difference of $v_{2}$ of proton and antiproton at various collision energies. Qualitatively they depict similar behavior. We also compare the data on light nuclei $v_{2}$ to various theoretical models such as blast-wave and coalescence. We then present a prediction of $v_{2}$ for ${ }^{3} \mathrm{He}$ and ${ }^{4} \mathrm{He}$ using coalescence and blast-wave models.
\end{abstract}

\section{Introduction}

The main goals of high energy heavy-ion collision experiments have primarily been to study the properties of Quark Gluon Plasma (QGP) and the other phase structures in the QCD phase diagram [1-11]. The energy densities created in these high energy collisions are similar to that found in the universe, microseconds after the Big Bang $[5-8,12-14]$. Subsequently, the universe cooled down to form nuclei. It is expected that high energy heavy-ion collisions will allow studying the production of light nuclei such as $d, t,{ }^{3} \mathrm{He}$, and their corresponding antinuclei. There are two possible production mechanisms for light (anti)nuclei. The first mechanism is thermal production of nucleusantinucleus pairs in elementary nucleon-nucleon or partonparton interactions [15-21]. However, due to their small ( few $\mathrm{MeV}$ ) binding energies, the directly produced nuclei or antinuclei are likely to break up in the medium before escaping. The second mechanism is via final state coalescence of produced (anti)nucleons or from transported nucleons [22-36]. The quark coalescence as a mechanism of hadron production at intermediate transverse momentum has been well established by studying the number of constituent quarks (NCQ) scaling for $v_{2}$ of identified hadrons measured at RHIC [37-45]. Light nuclei may also be produced via coalescence of quarks similar to the hadrons. But the nuclei formed via quark coalescence are highly unlikely to survive in the high temperature environment due to their small binding energies. In case of hadron formation by quark coalescence, the momentum space distribution of quarks is not directly measurable in experiments. However, in case of nucleon coalescence, momentum space distributions of both the constituents (nucleons) and the products (nuclei) are measurable in heavy-ion collision experiments. Therefore, measurements of $v_{2}$ of light nuclei provide a tool to understand the production mechanism of light nuclei and freeze-out properties at a later stage of the evolution. It also provides an excellent opportunity to understand the mechanism of coalescence at work in high energy heavy-ion collisions.

The production of light (anti)nuclei has been studied extensively at lower energies in Bevelac at LBNL [24, 46-49], AGS at RHIC [50-53], and SPS at CERN [54-58]. In the AGS 
experiments, it was found that the coalescence parameter $\left(B_{2}\right)$ is of similar magnitude for both $d$ and $\bar{d}$ indicating similar freeze-out hypersurface of nucleons and antinucleons. Furthermore, the dependence of $B_{2}$ on collision energy and $p_{T}$ indicated that light nuclei production is strongly influenced by the source volume and transverse expansion profile of the system $[58,59]$. In this paper, we review the results of elliptic flow of light nuclei measured at RHIC and LHC and discuss the possible mechanisms for the light nuclei production.

The paper is organized as follows. Section 2 briefly describes the definition of $v_{2}$, identification of light (anti)nuclei in the experiments and measurement of $v_{2}$ of light (anti)nuclei. In Section 3, we present the $v_{2}$ results for minimum bias collisions from various experiments. We also discuss the centrality dependence, difference between nuclei and antinuclei $v_{2}$, and the energy dependence of deuteron $v_{2}$. In the same section, we present the atomic mass number scaling and also compare the experimental results with various theoretical models. Finally in Section 4, we summarize our observations and discuss the main conclusions of this review.

\section{Experimental Method}

2.1. Elliptic Flow $\left(v_{2}\right)$. The azimuthal distribution of produced particles in heavy-ion collision can be expressed in terms of a Fourier series,

$$
\frac{d N}{d\left(\phi-\Psi_{r}\right)} \propto 1+\sum_{n} 2 v_{n} \cos \left[n\left(\phi-\Psi_{r}\right)\right]
$$

where $\phi$ is the azimuthal angle of produced particle, $\Psi_{r}$ is called the reaction plane angle, and the Fourier coefficients $v_{1}, v_{2}$, and so on are called flow coefficients [60]. $\Psi_{r}$ is defined as the angle between the impact parameter vector and the $x$ axis of the reference detector in the laboratory frame. Since it is impossible to measure the direction of impact parameter in heavy-ion collisions, a proxy of $\Psi_{r}$, namely, the event plane angle $\Psi_{n}$, is used for the flow analysis in heavy-ion collisions [61]. $v_{2}$ is measured with respect to the 2nd-order event plane angle $\Psi_{2}$ [61]. $\Psi_{2}$ is calculated using the azimuthal distribution of the produced particles. In an event with $N$ particles, the event plane angle $\Psi_{2}$ is defined as [61]

$$
\Psi_{2}=\frac{1}{2} \tan ^{-1}\left(\frac{Y_{2}}{X_{2}}\right) .
$$

$X_{2}$ and $Y_{2}$ are defined as

$$
\begin{aligned}
& X_{2}=\sum_{i=1}^{N} w_{i} \cos \left(2 \phi_{i}\right), \\
& Y_{2}=\sum_{i=1}^{N} w_{i} \sin \left(2 \phi_{i}\right),
\end{aligned}
$$

where $w_{i}$ are weights given to each particle to optimise the event plane resolution $[61,62]$. Usually the magnitude of particle transverse momentum $p_{T}$ is used as weights as $v_{2}$ increases with $p_{T}$. Special techniques are followed while calculating the event plane angle so that it does not contain the particle of interest whose $v_{2}$ is to be calculated (self-correlation) and also the nonflow effects (e.g., jets and short range correlations) are removed as much as possible $[41,42,61,63]$. Heavy-ion experiments use the $\eta$-subevent plane method to calculate the elliptic flow of identified hadrons as well as for light nuclei. In this method, each event is divided into two subevents in two different $\eta$-windows (e.g., positive and negative $\eta$ ). Then two subevent plane angles are calculated with the particles in each subevent. Each particle with a particular $\eta$ is then correlated with the subevent plane of the opposite $\eta$. This ensures that the particle of interest is not included in the calculation of event plane angle. A finite $\eta$ gap is applied between the two subevents to reduce short range correlations which does not originate from flow.

The distribution of the event plane angles should be isotropic in the laboratory frame for a azimuthally isotropic detector. If the distribution of the event plane angles is not flat in the laboratory frame (due to detector acceptance and/or detector inefficiency) then special techniques are applied to make the distribution uniform. The popular methods to make the $\Psi_{2}$ distribution uniform is the $\phi$-weight and recentering $[64,65]$. In the $\phi$-weight method, one takes the actual azimuthal distribution of the produced particle, averaged over large sample of events, and then uses inverse of this distribution as weights while calculating the correlation of the particles with the event plane angle $[64,65]$. In the recentering method, one subtracts $\left\langle X_{n}\right\rangle$ and $\left\langle Y_{n}\right\rangle$ from the event-by-event $X_{n}$ and $Y_{n}$, respectively, where $\left\langle X_{n}\right\rangle$ and $\left\langle Y_{n}\right\rangle$ denote the average of $X_{n}$ and $Y_{n}$ over a large sample of similar events. The main disadvantage of applying one of these methods is that it does not remove the contribution from higher flow harmonics. Therefore, another correction method known as the shift correction is used to remove the effects coming from higher flow harmonics. In this method, one fits the $\Psi_{2}$ distribution (after applying $\phi$-weight and/or recentering method) averaged over all events, with a Fourier function. The Fourier coefficients from this fit (obtained as fit parameters) are used to shift $\Psi_{2}$ of each event, to make the distribution uniform in the laboratory frame $[64,65]$.

Since the number of particles produced in heavy-ion collisions are finite, the calculated event plane angle $\Psi_{2}$ may not coincide with $\Psi_{r}$. For this reason, the measured $v_{2}^{\text {obs }}$ with respect to $\Psi_{2}$ is corrected with the event plane resolution factor $R_{2}$, where

$$
R_{2}=\left\langle\cos \left[2\left(\Psi_{2}-\Psi_{r}\right)\right]\right\rangle
$$

In order to calculate the event plane resolution, one calculates two subevent plane angles $\Psi_{2}^{a}$ and $\Psi_{2}^{b}$, where $a$ and $b$ correspond to two independent subevents. If the multiplicities of each subevent are approximately half of the full event plane, then the resolution of each of subevent plane can be calculated as $[60,61]$,

$$
\left\langle\cos \left[2\left(\Psi_{2}^{a}-\Psi_{r}\right)\right]\right\rangle=\sqrt{\left\langle\cos \left[2\left(\Psi_{2}^{a}-\Psi_{2}^{b}\right)\right]\right\rangle} .
$$


TABLE 1: Available measurements of light nuclei $v_{2}$.

\begin{tabular}{lccc}
\hline Experiment & Nuclei & $\sqrt{s_{\mathrm{NN}}}(\mathrm{GeV})$ & Centrality \\
\hline STAR [63] & $d, \bar{d}, t$, & $7.7,11.5,19.6,27$, & $0-80 \%, 0-30 \%, 30-80 \%$ \\
PHENIX [66] & ${ }^{3} \mathrm{He},{ }^{3} \overline{\mathrm{He}}$ & $39,62.4,200$ & $(0-10 \%, 10-40 \%, 40-80 \%$ in $200 \mathrm{GeV})$ \\
ALICE [67] & $d+\bar{d}$ & 200 & $0-20 \%, 20-60 \%$ \\
& $d+\bar{d}$ & 2760 & $0-5 \%, 5-10 \%, 10-20 \%, 20-30 \%, 30-40 \%, 40-50 \%$ \\
\hline
\end{tabular}

However, the full event plane resolution can be expressed as

$$
\begin{aligned}
& \left\langle\cos \left[2\left(\Psi_{2}-\Psi_{r}\right)\right]\right\rangle \\
& =\frac{\sqrt{\pi}}{2 \sqrt{2}} \chi_{2} \exp \left(-\frac{\chi_{2}^{2}}{4}\right) \times\left[I_{0}\left(\frac{\chi_{2}^{2}}{4}\right)+I_{2}\left(\frac{\chi_{2}^{2}}{4}\right)\right],
\end{aligned}
$$

where $\chi_{2}=v_{2} / \sigma$ and $I_{0}, I_{2}$ are modified Bessel functions $[60,61]$. The parameter $\sigma$ is inversely proportional to the square-root of $N$, the number of particles used to determine the event plane $[60,61]$. To calculate the resolution for full event plane $\left(\Psi_{2}\right)$, one has to solve (6) iteratively for the value of $\chi_{2}$ using the subevent plane resolution $\left(\left\langle\cos \left[2\left(\Psi_{2}^{a}-\Psi_{r}\right)\right]\right\rangle\right)$ which is calculated experimentally using (5). The $\chi_{2}$ value is then multiplied with $\sqrt{2}$ as $\chi_{2}$ is proportional to $\sqrt{N}$ and reused in (6) to calculate the resolution of the full event plane. In a case of very low magnitudes, the full event plane resolution can be approximately given as $[60,61]$

$$
\begin{aligned}
\left\langle\cos \left[2\left(\Psi_{2}-\Psi_{r}\right)\right]\right\rangle & =\sqrt{2}\left\langle\cos \left[2\left(\Psi_{2}^{a}-\Psi_{r}\right)\right]\right\rangle \\
& =\sqrt{2\left\langle\cos \left[2\left(\Psi_{2}^{a}-\Psi_{2}^{b}\right)\right]\right\rangle} .
\end{aligned}
$$

The procedure for calculating full and subevent plane resolutions using subevent plane angles and various approximations is discussed in detail in $[60,61]$.

2.2. Data on Light Nuclei. For this review, we have collected light nuclei $v_{2}$ data from the STAR [63] and PHENIX [66] experiments at RHIC and ALICE experiment at LHC [67]. Table 1 summarizes the measurement of light nuclei $v_{2}$ available till date.

2.3. Extraction of Light Nuclei $v_{2}$. In heavy-ion collisions, light nuclei are primarily identified by comparing the mean ionization energy loss per unit length $(\langle d E / d x\rangle)$ in the Time Projection Chamber (TPC) with that from the theoretical predictions $\left(d E /\left.d x\right|_{\text {theo }}\right)[41,42,63,67-71]$. Light nuclei are also identified via the time-of-flight measurement techniques using the Time-of-Flight (TOF) detector [66, 67, 71-74].

In the STAR experiment, to identify light nuclei using TPC, a variable $Z[63]$ is defined as

$$
Z=\log \left[\left.\frac{\langle d E / d x\rangle}{(d E / d x)}\right|_{\text {theo }}\right] .
$$

Then the light nuclei yields are extracted from these $Z$ distributions in differential $p_{T}$ and $\left(\phi-\Psi_{2}\right)$ bins either for minimum bias collisions or in selected centrality classes. The
$\left(\phi-\Psi_{2}\right)$ distribution is then fitted with a 2nd-order Fourier function; namely,

$$
\frac{d N}{d\left(\phi-\Psi_{2}\right)} \sim 1+2 v_{2} \cos \left(\phi-\Psi_{2}\right) .
$$

The Fourier coefficient $v_{2}$ is called elliptic flow and is extracted from the fit. As we discussed in the previous subsection this measured $v_{2}$ is then corrected with the event plane resolution factor $\left(R_{2}\right)[41,42,63]$.

In the ALICE experiment, light nuclei in the low $p_{T}$ region $(<1.0 \mathrm{GeV} / \mathrm{c}$ for $d, \bar{d})$ are identified by comparing the variance $\left(\sigma_{\langle d E / d x\rangle}\right)$ of the measured $\langle d E / d x\rangle$ in the TPC with the corresponding theoretical estimate $\left(d E /\left.d x\right|_{\text {theo }}\right)[67$, 71]. Light nuclei are considered identified if the measured $\langle d E / d x\rangle$ lies within $\pm 3 \sigma_{\langle d E / d x\rangle}$ of the $d E /\left.d x\right|_{\text {theo }}$. On the other hand, the light nuclei yield is extracted from the mass squared $\left(m_{\mathrm{TOF}}^{2}\right)$ distribution using the TOF detector. The mass of each particle is calculated using the time-of-flight $(t)$ from the TOF detector and the momentum (p) information from the TPC $[66,67,71]$. Both the ALICE and PHENIX experiments use the TOF detector to identify light nuclei at high $p_{T}(>1.0 \mathrm{GeV} / \mathrm{c})$. The mass of a particle can be calculated using the TOF detector as

$$
m_{\mathrm{TOF}}^{2}=\frac{\mathbf{p}^{2}}{c^{2}}\left(\frac{c^{2} t^{2}}{L^{2}}-1\right),
$$

where the track length $L$ and momentum $\mathbf{p}$ are determined with the tracking detectors placed inside magnetic field [44, $66,67,71]$. After getting $m^{2}$ for each particle, a selection cut is implemented to reject tracks which have their $m^{2}$ several $\sigma$ away from the true $m^{2}$ value of the light nuclei, as done in the STAR experiment [63]. The ALICE experiment, on the other hand, defines a quantity $\Delta m$ such that $\Delta m=$ $m_{\mathrm{TOF}}-m_{\text {nucl }}$, where $m_{\text {nucl }}$ is the mass of the light nuclei under study. The distribution of $\Delta m$ is then fitted with a Gaussian + exponential function for signal and an exponential function for the background [67]. Then $v_{2}$ of light nuclei is calculated by fitting $v_{2}(\Delta m)$ with the weighted function,

$$
\begin{aligned}
v_{2}^{\text {Tot }}(\Delta m)= & v_{2}^{\operatorname{Sig}}(\Delta m) \frac{N^{\text {Sig }}}{N^{\text {Tot }}}(\Delta m) \\
& +v_{2}^{\text {Bkg }}(\Delta m) \frac{N^{\mathrm{Bkg}}}{N^{\mathrm{Tot}}}(\Delta m),
\end{aligned}
$$

where the total measured $v_{2}^{\text {Tot }}$ is the weighted sum of that from the signal $\left(v_{2}^{\mathrm{Sig}}\right)$ and background $\left(v_{2}^{\mathrm{Bkg}}\right) \cdot v_{2}^{\text {Tot }}$ of the candidate 

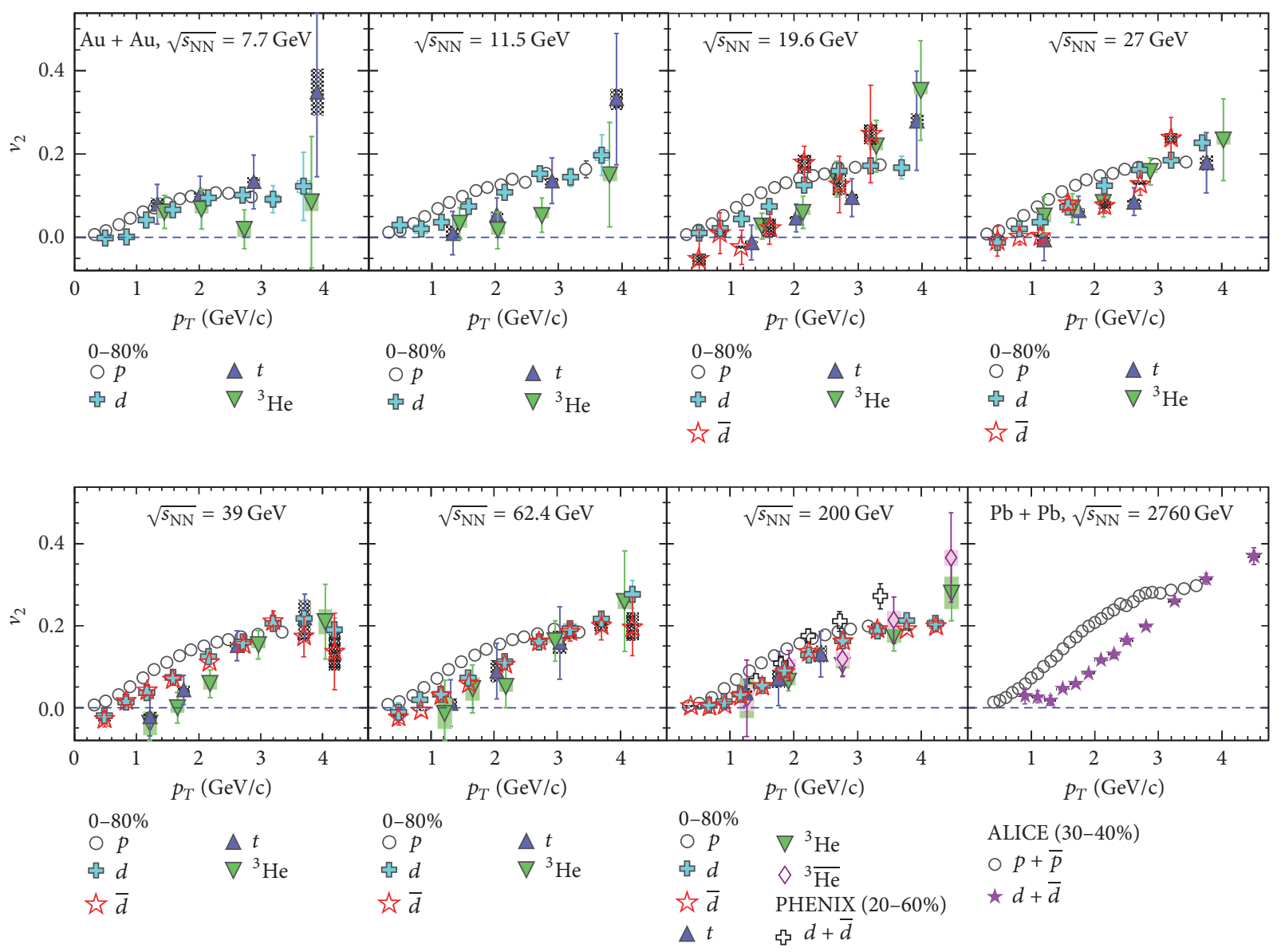

FIgure 1: Midrapidity $v_{2}\left(p_{T}\right)$ for light nuclei $\left(d, \bar{d}, t,{ }^{3} \mathrm{He}\right.$, and $\left.{ }^{3} \overline{\mathrm{He}}\right)$ in $0-80 \%, 20-60 \%$, and $30-40 \%$ centrality from STAR, PHENIX, and ALICE, respectively. Proton $v_{2}\left(p_{T}\right)$ is also shown as open circles $[41-44,75]$ for comparison. Lines and boxes at each marker corresponds to statistical and systematic errors, respectively.

particles are calculated using the scalar product method and corrected for the event plane resolution [67].

The PHENIX experiment calculates charged average $v_{2}$ of (anti) deuterons as

$$
v_{2}=\frac{\left\langle\cos \left(2\left(\phi-\Psi_{2}\right)\right)\right\rangle}{R_{2}} .
$$

The quantity $R_{2}=\left\langle\cos \left(2\left(\Psi_{2}-\Psi_{r}\right)\right)\right\rangle$ can readily be identified as the resolution of the event plane angle [66]. The resolution of full event plane $\Psi_{2}$ is calculated with subevent planes $\left(\Psi_{2}^{a}, \Psi_{2}^{b}\right)$ estimated using two Beam-Beam Counter (BBC) detectors $[44,66]$. The detailed procedure of calculating the full event plane resolution from subevents is already mentioned in the previous subsection. The large $\eta$ gap between the central TOF and the BBCs $(\Delta \eta>2.75)$ reduces the effects of nonflow significantly $[44,66]$. The nuclei $v_{2}$ calculated in PHENIX are also corrected for the contribution coming from backgrounds, mainly consisting of misidentification of other particles (e.g., protons) as nuclei.
A $p_{T}$ dependent correction factor was applied on the total $v_{2}$ (referred to as $v_{2}^{\text {Sig }+\mathrm{Bkg}}\left(p_{T}\right)$ ) such that

$$
v_{2}^{d(\bar{d})}\left(p_{T}\right)=\frac{\left[v_{2}^{\text {Sig+Bkg }}\left(p_{T}\right)-(1-R) v_{2}^{\mathrm{Bkg}}\left(p_{T}\right)\right]}{R},
$$

where $v_{2}^{\mathrm{Sig}+\mathrm{Bkg}}\left(p_{T}\right)$ is the measured $v_{2}$ for $d(\bar{d})+$ background at a given $p_{T}, v_{2}^{d(\bar{d})}$ is the corrected $v_{2}$ of $d(\bar{d})$, and $R$ is the ratio of signal and signal + background.

\section{Results and Discussion}

3.1. General Aspects of Light Nuclei $v_{2}$. Figure 1 shows the energy dependence of light (anti)nuclei $v_{2}$ for $\sqrt{s_{\mathrm{NN}}}=7.7$, $11.5,19.6,27,39,62.4,200$, and $2760 \mathrm{GeV}$. The panels are arranged by increasing energy from left to right and top to bottom. The $p_{T}$ dependence of $v_{2}$ of $d, \bar{d}, t,{ }^{3} \mathrm{He}$, and ${ }^{3} \overline{\mathrm{He}}$ is shown for $0-80 \%$ centrality in STAR, 20-60\% centrality in PHENIX, and 30-40\% centrality in ALICE. Since PHENIX and ALICE do not have measurements in the minimum bias collisions, we only show the results for mid-central collisions. The data points of PHENIX and ALICE correspond to 


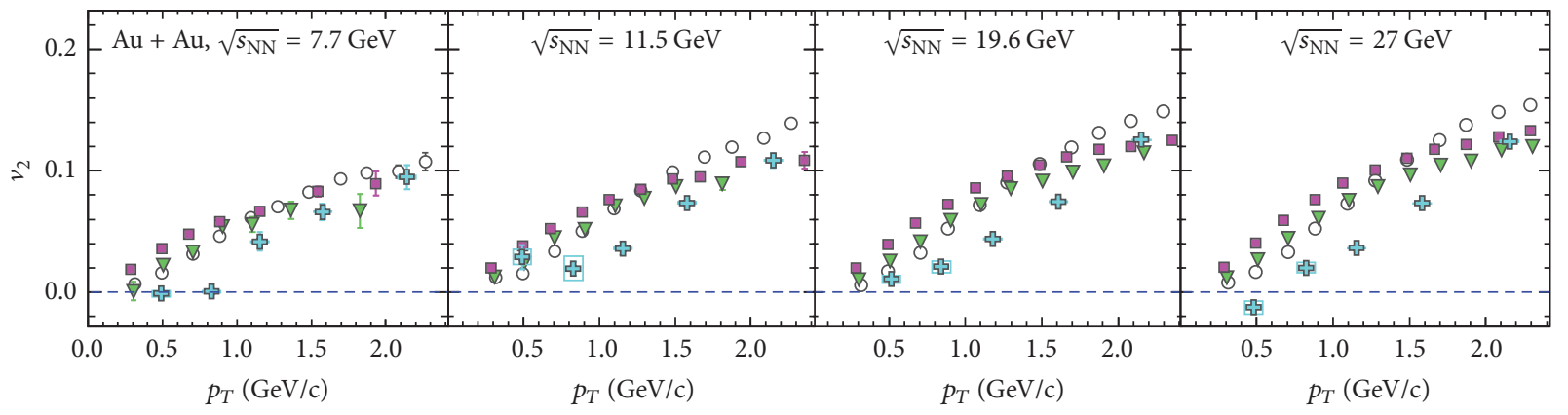

\begin{tabular}{|c|c|c|c|c|c|c|}
\hline $\begin{array}{l}\text { STAR } \\
\pi^{+}(\pi)\end{array}$ & $\circ p$ & $\begin{array}{l}\text { STAR } \\
\pi^{+}(\pi)\end{array}$ & $\circ p$ & $\begin{array}{l}\text { STAR } \\
\text { - } \pi^{+}(\pi)\end{array}$ & $\circ p$ & $\begin{array}{l}\text { STAR } \\
\pi^{+}(\pi)\end{array}$ \\
\hline$\nabla K_{s}^{0}(K)$ & $\nLeftarrow d$ & $\nabla K_{s}^{0}(K)$ & $\Leftrightarrow d$ & $\nabla K_{s}^{0}(K)$ & њ $d$ & $\nabla K_{s}^{0}(K)$ \\
\hline
\end{tabular}

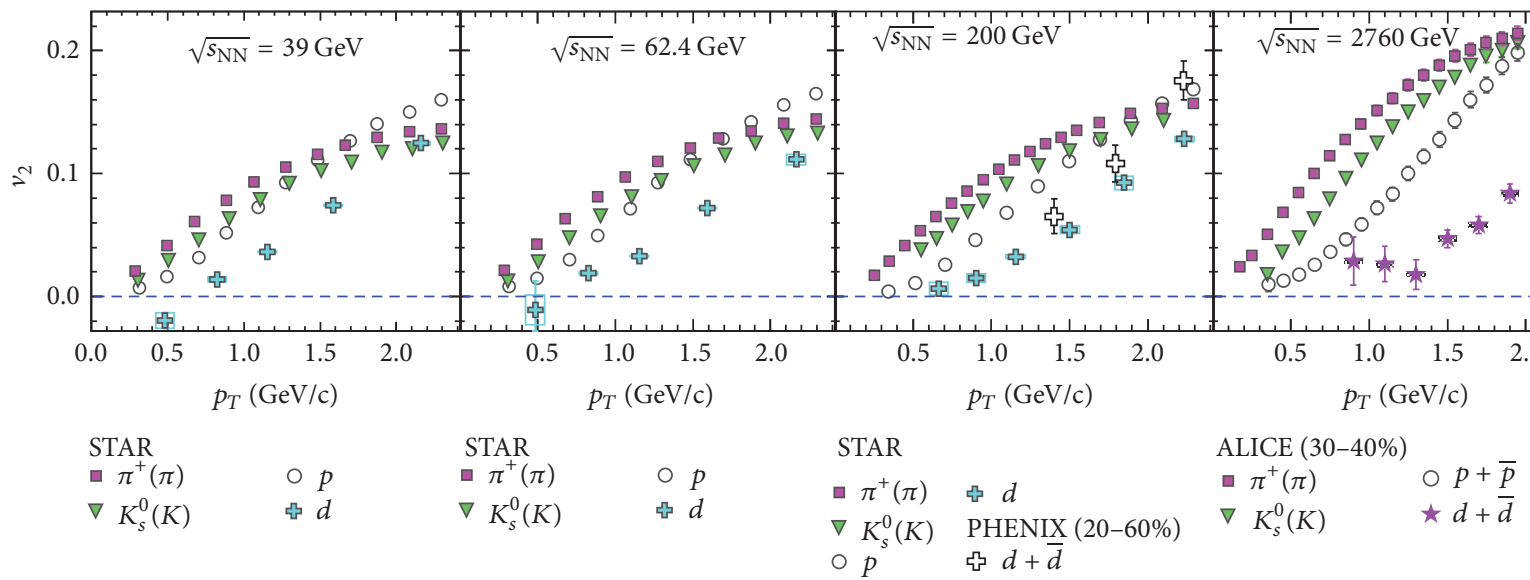

Figure 2: Midrapidity $v_{2}\left(p_{T}\right)$ for $\pi^{+}$(squares), $K_{s}^{0}(K$ in $\mathrm{Pb}+\mathrm{Pb}$ ) (triangles), $p$ (open circles), and $d$ (crosses) in $0-80 \%, 20-60 \%$, and $30-40 \%$ centrality from STAR, PHENIX, and ALICE, respectively.

inclusive $d+\bar{d} v_{2}$. The general trend of nuclei $v_{2}$ of all species is the same: it increases with increasing $p_{T}$. The slight difference of $v_{2}$ between STAR and PHENIX is due to the difference in centrality ranges. The centrality range for PHENIX is $20-60 \%$ and that for STAR is $0-80 \%$.

From the trend in Figure 1 it seems that light nuclei $v_{2}$ show mass ordering; that is, heavier particles have smaller $v_{2}$ value compared to lighter ones, similar to $v_{2}$ of identified particles $[41,42,44,75]$. In order to see the mass ordering effect more clearly, we restrict the $x$-axis range to $2.5 \mathrm{GeV} / \mathrm{c}$ and compare $v_{2}$ of $d$ with $v_{2}$ of identified particles such as $\pi^{+}$, $K_{s}^{0}(K$ in $\mathrm{Pb}+\mathrm{Pb})$, and $p$ as shown in Figure 2. We see that $d v_{2}$ at all collision energies is lower than $v_{2}$ of the identified hadrons at a fixed value of $p_{T}$. Although mass ordering is a theoretical expectation from the hydrodynamical approach to heavy-ion collisions [76], coalescence formalism for light nuclei can also give rise to this effect. Recent studies using AMPT and VISHNU hybrid model suggest that mass ordering is also expected from transport approach to heavy-ion collisions [77-79]. $v_{2}$ of light nuclei is negative for some collision energies as shown in Figure 1. This negative $v_{2}$ is expected to be the outcome of strong radial flow in heavy-ion collisions [80].

In order to study the energy dependence of light nuclei $v_{2}$, we compare the deuteron $v_{2}$ from collision energy $\sqrt{s_{\mathrm{NN}}}=$
$7.7 \mathrm{GeV}$ to $2760 \mathrm{GeV}$ as shown in Figure 3. The deuteron $v_{2}\left(p_{T}\right)$ shows energy dependence prominently for high $p_{T}$ $\left(p_{T}>2.4 \mathrm{GeV} / \mathrm{c}\right)$, where $v_{2}$ is highest for top collision energy $\left(\sqrt{s_{\mathrm{NN}}}=2760 \mathrm{GeV}\right)$ and gradually decreases with decreasing collision energy. This energy dependent trend of light nuclei $v_{2}$ is similar to the energy dependence of identified hadron $v_{2}$ where $v_{2}\left(p_{T}\right)$ also decreases with decreasing collision energy $[41,42]$.

The STAR experiment has measured the difference of nuclei $(d)$ and antinuclei $(\bar{d}) v_{2}$ for collision energies $\sqrt{s_{\mathrm{NN}}}=$ 19.6, 27, 39, 62.4, and $200 \mathrm{GeV}$ [63]. Figure 4 shows the difference of $d$ and $\bar{d} v_{2}$ as a function of collision energy. For comparison, the difference of proton and antiproton $v_{2}$ is also shown [41, 42]. We observe that the difference of $d$ and $\bar{d} v_{2}$ remains positive for $\sqrt{s_{\mathrm{NN}}}=7.7-39 \mathrm{GeV}$. However, for $\sqrt{s_{\mathrm{NN}}} \geq 62.4 \mathrm{GeV}$ the difference of $d$ and $\bar{d} v_{2}$ is almost zero. The difference of $d$ and $\bar{d} v_{2}$ qualitatively follows the same trend as seen for difference of $p$ and $\bar{p} v_{2}[41,42]$. It is easy to infer from simple coalescence model that light (anti)nuclei formed via coalescence of (anti)nucleons will also reflect similar difference in $v_{2}$ as the constituent nucleon and antinucleon. The difference in $v_{2}$ between particles and their antiparticles has been attributed to the chiral magnetic effect in finite baryon-density matter [81], different $v_{2}$ of produced 


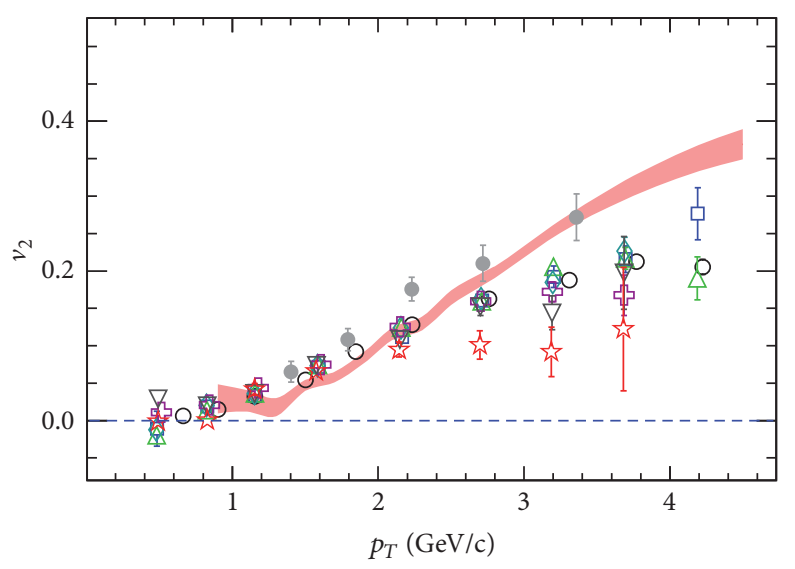

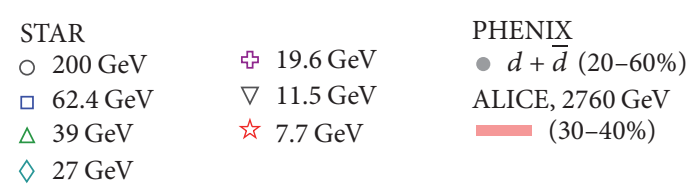

FIGURE 3: Energy dependence of midrapidity $v_{2}\left(p_{T}\right)$ of $d$ for minimum bias (30-40\% for ALICE) collisions.

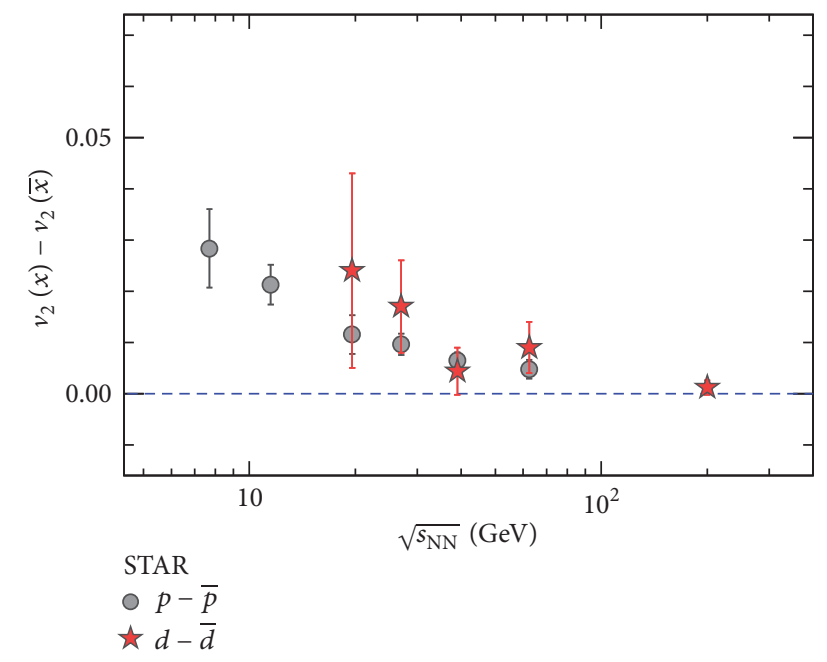

FIGURE 4: Difference of $d$ and $\bar{d} v_{2}\left(p_{T}\right)$ as a function of collision energy for minimum bias $\mathrm{Au}+\mathrm{Au}$ collisions in STAR.

and transported particles [82], different rapidity distributions for quarks and antiquarks [83], the conservation of baryon number, strangeness, and isospin [84], and different meanfield potentials acting on particles and their antiparticles [85-88].

The centrality dependence of light nuclei $v_{2}$ measured by the STAR and ALICE is shown in Figure 5. STAR has measured $d$ and $\bar{d} v_{2}$ in two different centrality ranges, namely, $0-30 \%$ and $30-80 \%$ for collision energies below $\sqrt{s_{\mathrm{NN}}}=200 \mathrm{GeV}$. In case of $\sqrt{s_{\mathrm{NN}}}=200 \mathrm{GeV}$, the light nuclei $v_{2}$ are measured in three different centrality ranges, namely, $0-10 \%$ (central), $10-40 \%$ (mid-central), and $40-80 \%$ (peripheral) as high statistics data were available. ALICE has measured inclusive $d+\bar{d} v_{2}$ in 6 different centrality ranges, namely, $0-5 \%, 5-10 \%, 10-20 \%, 20-30 \%, 30-40 \%$, and $40-50 \%$. We only present the results from $0-5 \%, 20-30 \%$, and $40-50 \%$ centrality from ALICE as shown in Figure 5. $v_{2}$ of $d$ shows strong centrality dependence for all collision energies studied in the STAR experiment. We observe that more central events have lower $v_{2}$ compared to peripheral events. $\bar{d}$ shows the same trend as $d$ for collision energies down to $\sqrt{s_{\mathrm{NN}}}=27 \mathrm{GeV}$.

The STAR experiment could not study centrality dependence of $\bar{d}$ below $\sqrt{s_{\mathrm{NN}}}=27 \mathrm{GeV}$ due to limited event statistics [63]. Comparing the centrality dependence of $d(\bar{d}) v_{2}$ from STAR and ALICE we can see that both experiments show strong centrality dependence of light nuclei $v_{2}$. The centrality dependence of light nuclei $v_{2}$ is analogous to the centrality dependence observed for identified nucleon $(p, \bar{p})$ $v_{2}[89,90]$.

3.2. Mass Number Scaling and Model Comparison. It is expected from the formulations of coalescence model that if light nuclei are formed via the coalescence of nucleons then the elliptic flow of light nuclei, when divided by atomic mass number $(A)$, should scale with the elliptic flow of nucleons $[91,92]$. Therefore, we expect that the light (anti)nuclei $v_{2}$ divided by $A$ should scale with $p(\bar{p}) v_{2}$. Here, we essentially assume that $v_{2}$ of (anti)proton and (anti)neutron are the same as expected from the observed NCQ scaling of identified particle $v_{2}[41,42]$. Figure 6 shows the atomic mass number scaling of light nuclei $v_{2}$ from STAR, PHENIX, and ALICE experiments. Since ALICE does not have results in minimum bias events, we used both $p+\bar{p}$ and $d+\bar{d} v_{2}$ from $30-40 \%$ centrality range. We observe that light nuclei $v_{2}$ from STAR and PHENIX show atomic mass number scaling up to $p_{T} / A \sim 1.5 \mathrm{GeV} / \mathrm{c}$. However, deviation of the scaling of the order of $20 \%$ is observed for $d+\bar{d} v_{2}$ from ALICE. The scaling of light (anti)nuclei $v_{2}$ with (anti)proton $v_{2}$ suggests 

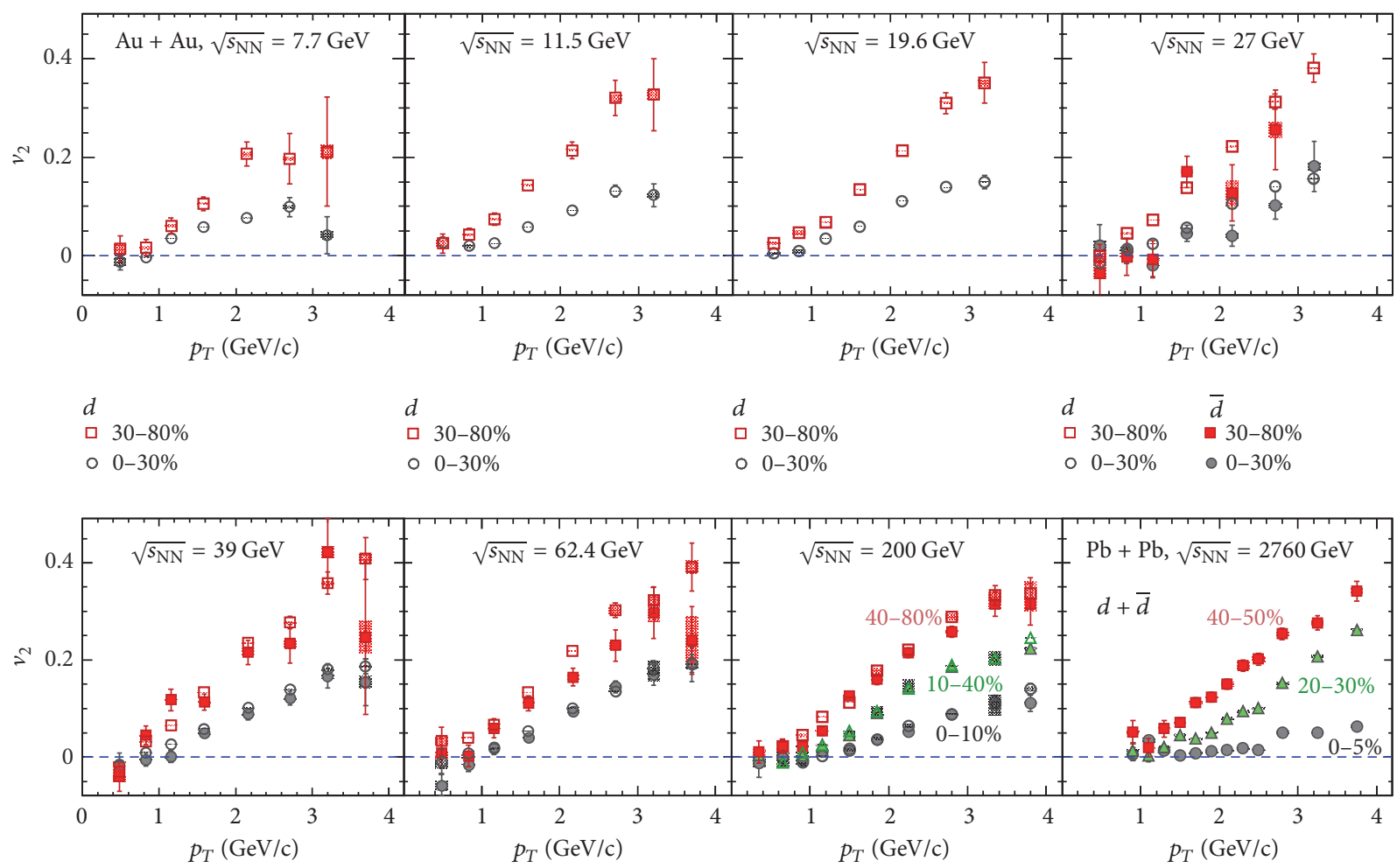

\begin{tabular}{|c|c|c|c|}
\hline & & & \\
\hline ㅁ 30-80\% & - $30-80 \%$ & ㅁ 30-80\% & - $30-80 \%$ \\
\hline ○ $0-30 \%$ & ㅇ $0-30 \%$ & ○ $0-30 \%$ & ค $0-30 \%$ \\
\hline
\end{tabular}

FIGURE 5: Centrality dependence of $v_{2}$ of $d(\bar{d})$ as a function of $p_{T}$.

that light (anti)nuclei might have formed via coalescence of (anti)nucleons at a later stage of the evolution at RHIC energies for $p_{T} / A$ up to $1.5 \mathrm{GeV} / \mathrm{c}$ [22-32]. However, this simple picture of coalescence may not be holding for ALICE experiment at LHC energies. On the contrary, there is another method to produce light nuclei, for example, by thermal production in which it is assumed that light nuclei are produced thermally like any other primary particles [17-21]. Various thermal model studies have successfully reproduced the different ratios of produced particles as well as light nuclei in heavy-ion collisions [17-21].

In order to investigate the success of these models, both STAR and ALICE have compared the elliptic flow of light nuclei with the predictions from blast-wave models [63, 67]. Figure 7 shows the $v_{2}$ of light nuclei predicted from blastwave model using the parameters obtained from fits to the identified particles $v_{2}[67,93]$. We observe that blastwave model reproduces $v_{2}$ of light nuclei from STAR with moderate success except for low $p_{T}(<1.0 \mathrm{GeV} / \mathrm{c})$, where $v_{2}$ of $d(\bar{d})$ are underpredicted for all collision energies. However, the blast-wave model seems to successfully reproduce the $d+\bar{d} v_{2}$ from ALICE. The low relative production of light nuclei compared to identified nucleons at RHIC collisions energies supports the procedure of light nuclei production via coalescence mechanism [22-32]. However, the success of blast-wave model in reproducing the nuclei $v_{2}$ at LHC and moderate success at RHIC suggest that the light nuclei production is also supported by thermal process [17-21]. The light nuclei production in general might be a more complicated coalescence process, for example, coalescence of nucleons in the local rest frame of the fluid cell. This scenario might give rise to deviations from simple $A$ scaling [63].

At RHIC energies the light nuclei $v_{2}$ have been compared with results from a hybrid AMPT + coalescence model [63]. A Multiphase Transport (AMPT) model is an event generator with Glauber Monte Carlo initial state [94]. The AMPT model includes Zhang's Partonic Cascade (ZPC) model for initial partonic interactions and A Relativistic Transport (ART) model for later hadronic interactions [94]. The nucleon phase-space information from the AMPT model is fed to the coalescence model to generate light nuclei $[63,95]$. Figure 8 shows the light nuclei $v_{2}$ from the coalescence model and compared to the data. The coalescence model prediction for $d+\bar{d}$ in $\mathrm{Pb}+\mathrm{Pb}$ collisions at $\sqrt{s_{\mathrm{NN}}}=2760 \mathrm{GeV}$ is taken from [96]. The coalescence model fairly reproduces the measurement from data for all collision energies except for the lowest energy $\sqrt{s_{\mathrm{NN}}}=7.7 \mathrm{GeV}$. The AMPT model generates nucleon $v_{2}$ from both partonic and hadronic interactions for all the collision energies presented. However, increased hadronic interactions compared to partonic, at lowest collision energies, are not implemented in the AMPT + coalescence model. This could be the reason behind the 

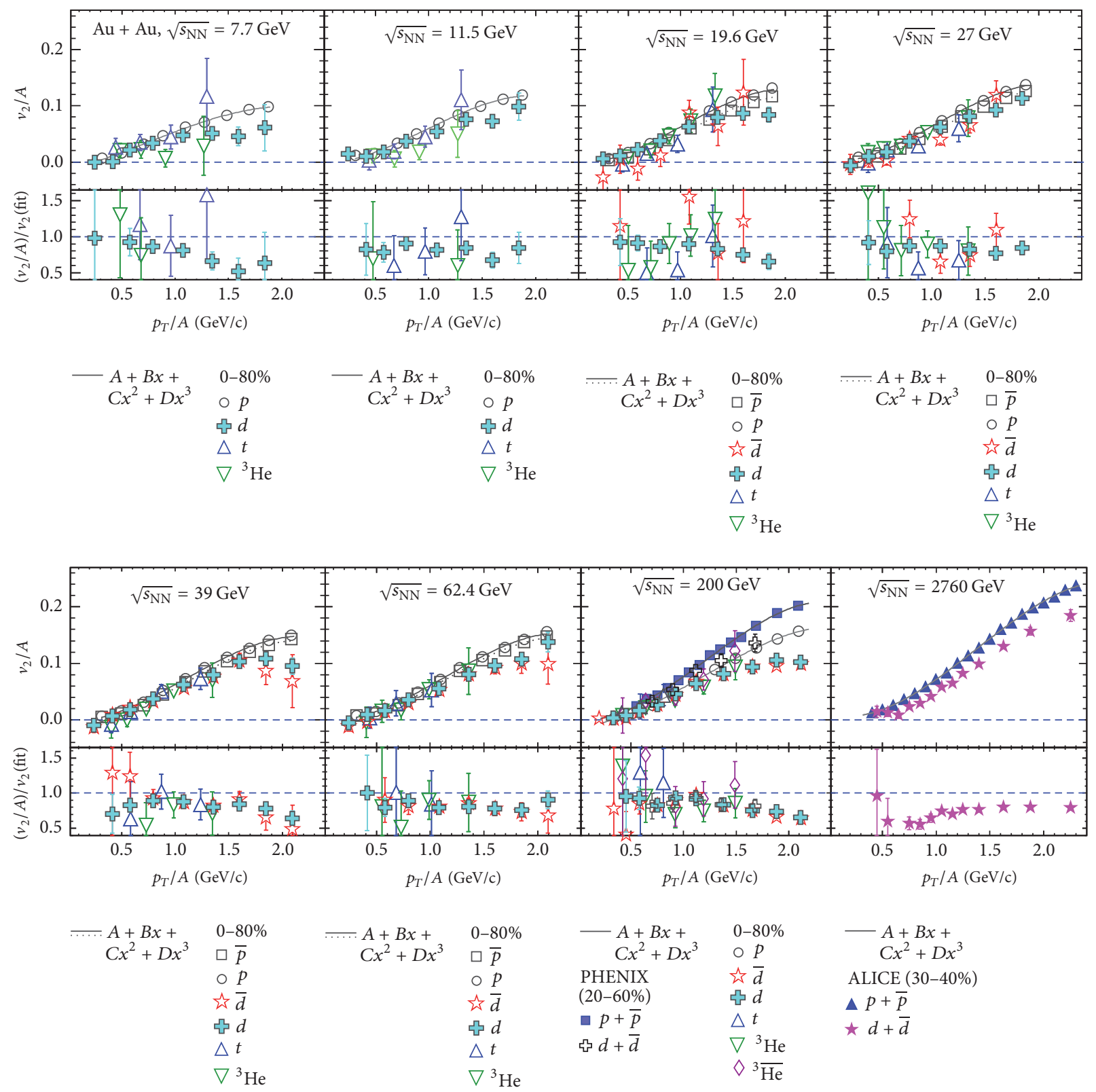

Figure 6: Atomic mass number scaling $v_{2} / A$ of light nuclei as a function of $p_{T} / A$ for STAR (0-80\%), PHENIX (20-60\%), and ALICE (30-40\%).

deviation of the data from the model predictions at lowest collision energy [41, 42].

We have performed simultaneous fit to the $v_{2}$ and $p_{T}$ spectra of identified hadrons + light nuclei using the same blast-wave model as used in $[67,75]$. The simultaneous fit of $v_{2}$ and $p_{T}$ spectra for measurements from the PHENIX and the ALICE experiment is shown in Figure 9. We find that the inclusion of light nuclei results to the fit does not change the fit results compared to the blast-wave fit performed only on identified hadron $v_{2}$ and $p_{T}$ spectra. This indicates that the light nuclei $v_{2}$ and $p_{T}$ spectra are well described by the blastwave model.
3.3. Model Prediction of ${ }^{3} \mathrm{He}$ and ${ }^{4} \mathrm{He} v_{2}$. We have predicted $v_{2}$ of ${ }^{3} \mathrm{He}$ and ${ }^{4} \mathrm{He}$ using the simple coalescence and blastwave model. Since protons and neutrons have similar masses and the same number of constituent quarks, they should exhibit similar collective behavior and, hence, similar magnitude of $v_{2}$. Therefore, we parametrize the elliptic flow of $p+\bar{p} v_{2}$ using the fit formula [97],

$$
f_{v_{2}\left(p_{T}\right)}(n)=\frac{a n}{1+e^{-\left(p_{T} / n-b\right) / c}}-d n,
$$

where $a, b, c$, and $d$ are fit parameters and $n$ is the constituent quark number of the particle [97]. The fit to $p+\bar{p} v_{2}$ (solid 


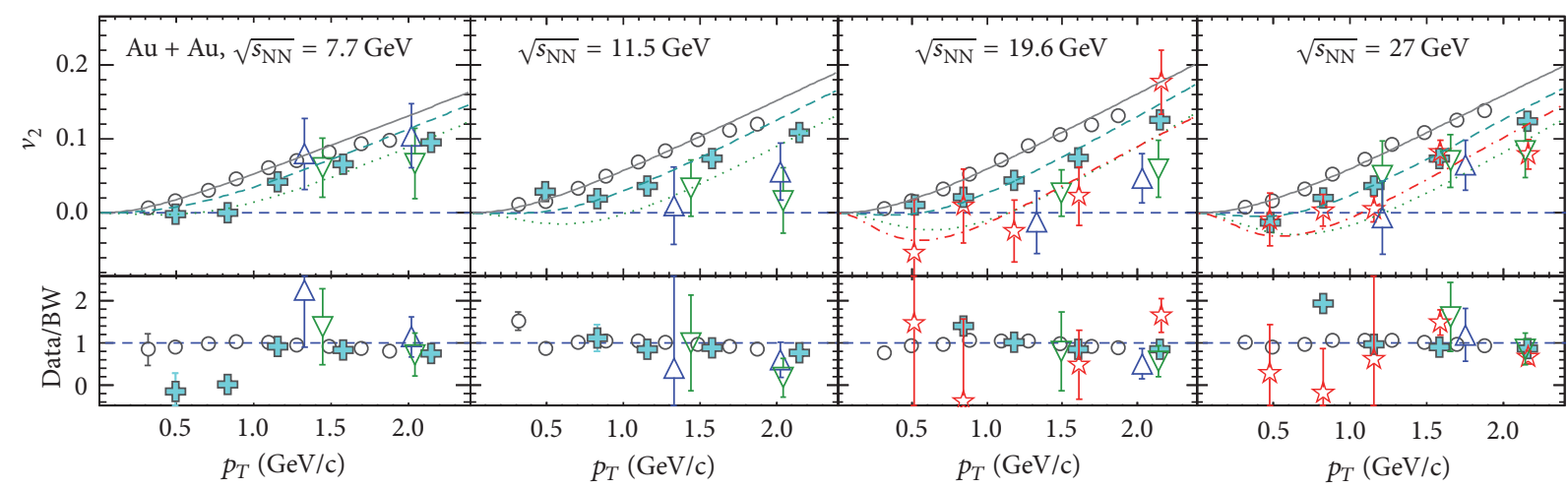

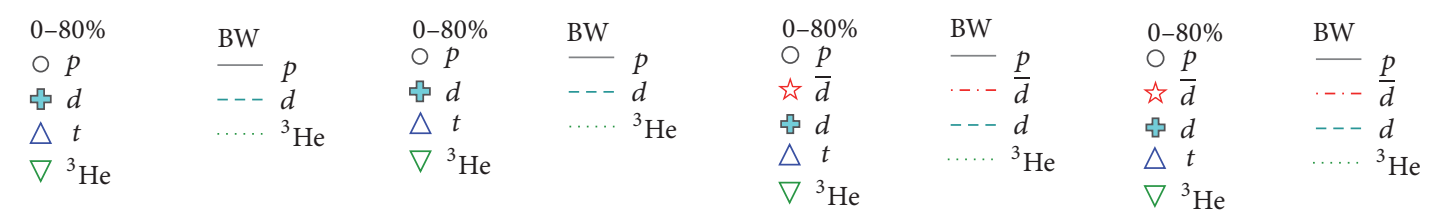

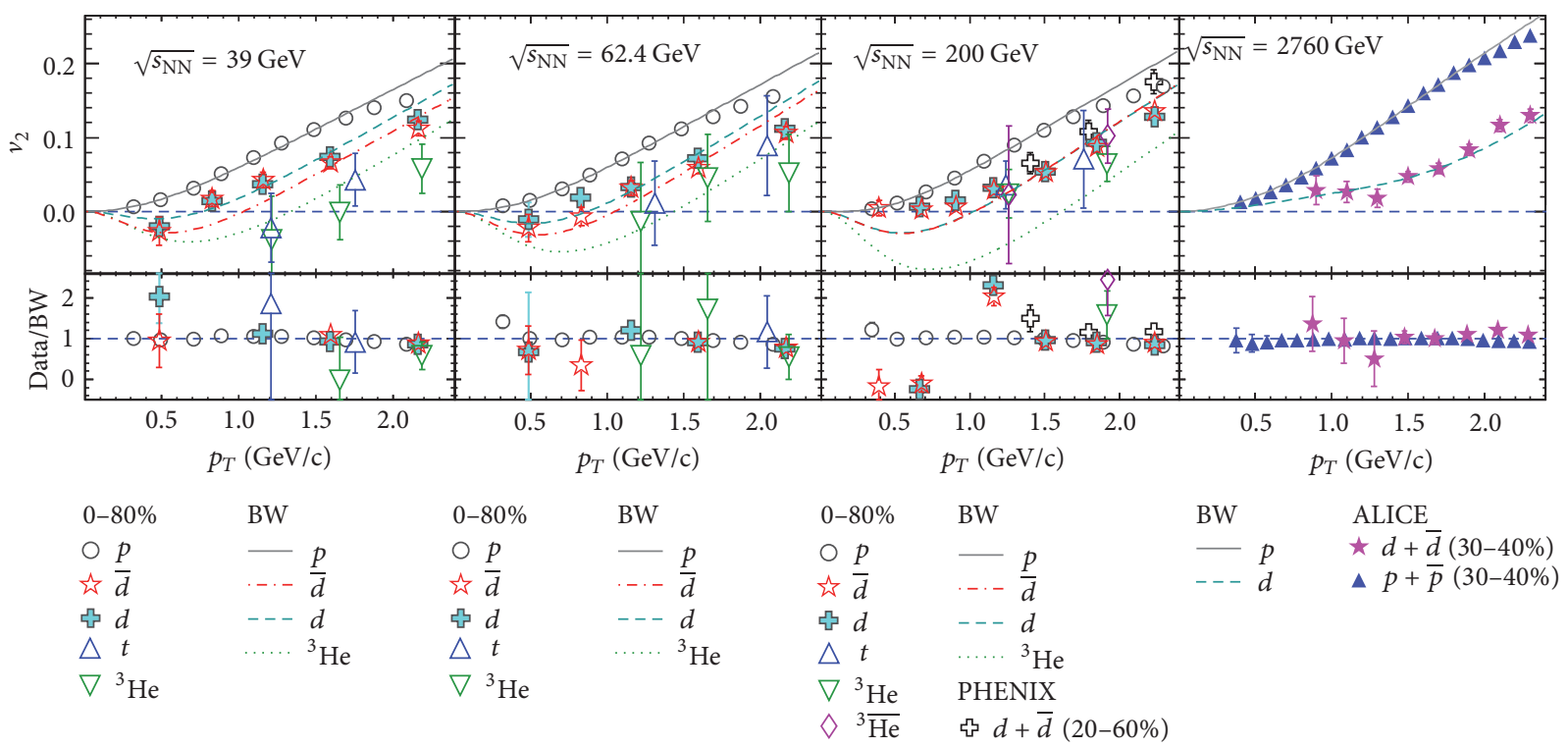

FIGURE 7: Light nuclei $v_{2}$ as a function of $p_{T}$ from blast-wave model (lines). For comparison, $p+\bar{p} v_{2}$ is also shown. Marker for STAR corresponds to $0-80 \%$, PHENIX corresponds to $20-60 \%$, and ALICE corresponds to $30-40 \%$ central events.

lines) from the PHENIX and ALICE experiment is shown in Figures 10(a) and 10(b), respectively. Assuming similar magnitude of neutron $v_{2}$ as that of proton, we then predict $v_{2}$ of ${ }^{3} \mathrm{He}$ and ${ }^{4} \mathrm{He}$ as

$$
\begin{aligned}
& v_{2}\left(p_{T}\right)_{{ }^{3} \mathrm{He}} \approx 3 v_{2}\left(\frac{p_{T}}{3}\right)_{p}, \\
& v_{2}\left(p_{T}\right)_{4} \mathrm{He}
\end{aligned}
$$

This simplified coalescence model prediction of ${ }^{3} \mathrm{He}$ and ${ }^{4} \mathrm{He} v_{2}$ is shown in Figures 10(a) and 10(b) as blue (dotted and dashed) lines. For comparison, the blast-wave model predicted $v_{2}$ of ${ }^{3} \mathrm{He}$ and ${ }^{4} \mathrm{He}$ from the fit parameters obtained in Figure 9 are also shown in red (dotted and dashed) lines. We observe characteristic difference in the prediction of ${ }^{3} \mathrm{He}$ and ${ }^{4} \mathrm{He} v_{2}$ from the coalescence and the blast-wave model. As one expects from the mass ordering effect of blast-wave model, $v_{2}$ of ${ }^{3} \mathrm{He}$ and ${ }^{4} \mathrm{He}$ are predicted to be almost zero in the intermediate $p_{T}$ range $\left(1.0<p_{T}<2.5 \mathrm{GeV} / \mathrm{c}\right)$. On the other hand, the simple coalescence model predicts orders of magnitude higher $v_{2}$ compared to blast-wave for both ${ }^{3} \mathrm{He}$ and ${ }^{4} \mathrm{He}$ in the same $p_{T}$ range. Hence, experimental measurements of ${ }^{3} \mathrm{He}$ and ${ }^{4} \mathrm{He} v_{2}$ in future would significantly improve our knowledge on the mechanisms of light nuclei formation in heavy-ion collisions [71, 98-100].

\section{Summary and Conclusions}

We have presented a review of elliptic flow $v_{2}$ of light nuclei $\left(d, t\right.$, and $\left.{ }^{3} \mathrm{He}\right)$ and antinuclei $\left(\bar{d}\right.$ and $\left.{ }^{3} \overline{\mathrm{He}}\right)$ from STAR experiment and inclusive $d+\bar{d} v_{2}$ from PHENIX at RHIC 

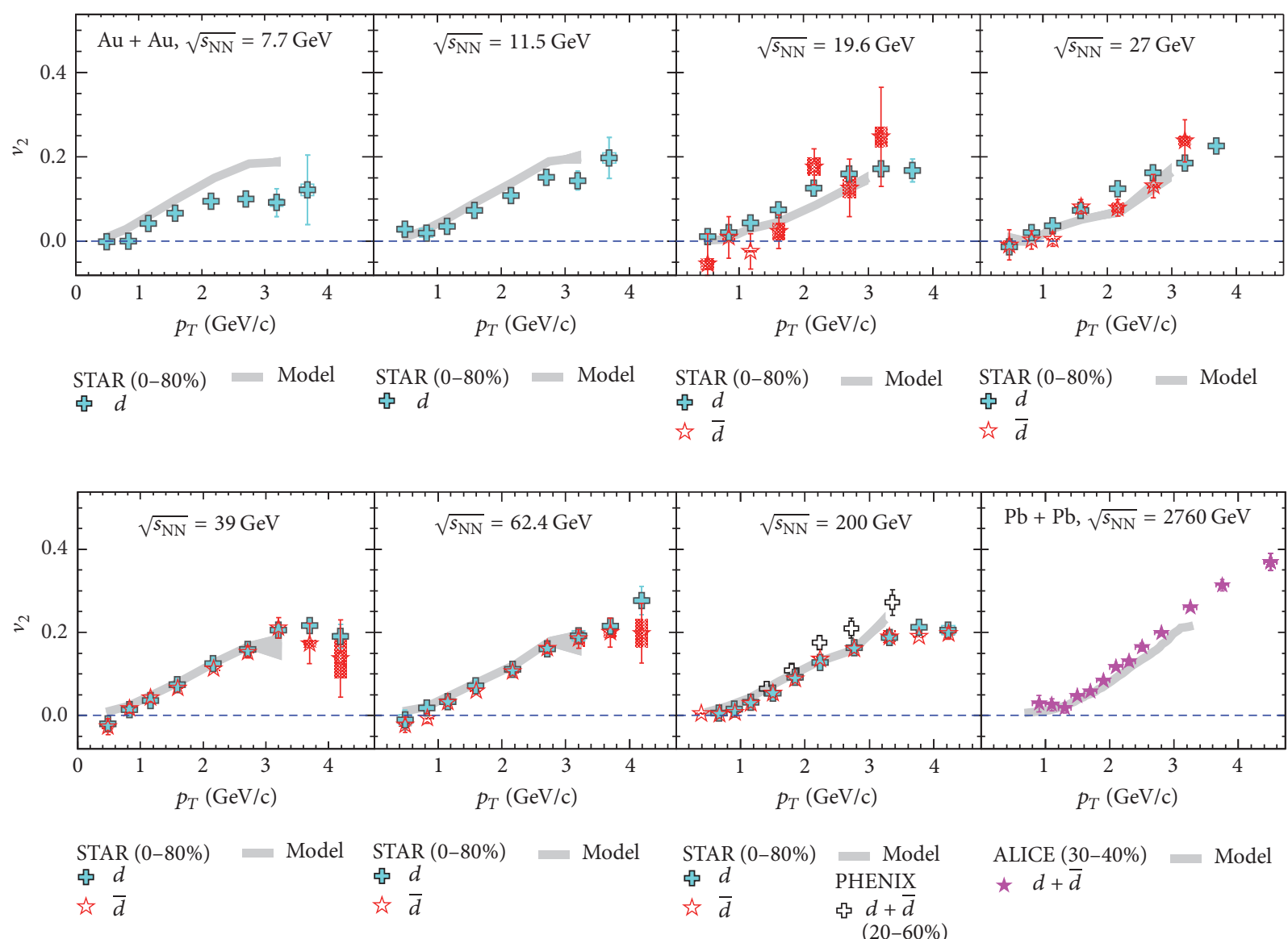

FIGURE 8: Light nuclei $v_{2}$ as a function of $p_{T}$ from AMPT + coalescence model (solid lines). Marker for STAR experiment corresponds to 0-80\%, PHENIX corresponds to 20-60\%, and ALICE corresponds to $30-40 \%$ central events.

and ALICE at LHC. Similar to identified hadrons, the light nuclei $v_{2}$ show a monotonic rise with increasing $p_{T}$ and mass ordering at low $p_{T}$ for all measured collision energies. The beam energy dependence of $d v_{2}$ is small at intermediate $p_{T}$ and only prominent at high $p_{T}$, which is similar to the trend as observed for the charged hadron $v_{2}$. The $v_{2}$ of nuclei and antinuclei are of similar magnitude for top collision energies at RHIC but at lower collision energies; the difference in $v_{2}$ between nuclei and antinuclei qualitatively follows the difference in proton and antiproton $v_{2}$. The centrality dependence of light (anti)nuclei $v_{2}\left(p_{T}\right)$ is similar to that of identified hadrons $v_{2}\left(p_{T}\right)$.

Light (anti)nuclei $v_{2}$ is found to follow the atomic mass number $(A)$ scaling for almost all collision energies at RHIC suggesting coalescence as the underlying process for the light nuclei production in heavy-ion collisions. However, a deviation from mass number scaling at the level of $20 \%$ is observed at LHC. This indicates that a simple coalescence process may not be the only underlying mechanism for light nuclei production. Furthermore, a transport-plus-coalescence model study is found to approximately reproduce the light nuclei $v_{2}$ measured at RHIC and LHC. The agreement of coalescence model with the data from PHENIX and STAR is imperceptibly better than the blast-wave model. However, at the LHC energy, the light nuclei $v_{2}$ are better described by blastwave model rather than the simple coalescence model. The coalescence mechanism, intuitively, should be the prominent process of light nuclei production. However, the breaking of mass scaling at LHC energy and success of blast-wave model prevent us from drawing any definitive conclusion on the light nuclei production mechanism.

We observed orders of magnitude difference in ${ }^{3} \mathrm{He}$ and ${ }^{4} \mathrm{He} v_{2}$ as predicted by blast-wave and coalescence model. The blast-wave model predicts almost zero $v_{2}$ for ${ }^{3} \mathrm{He}$ and ${ }^{4} \mathrm{He}$ up to $p_{T}=2.5 \mathrm{GeV} / \mathrm{c}$, whereas the coalescence model predicts significant $v_{2}$ for ${ }^{3} \mathrm{He}$ and ${ }^{4} \mathrm{He}$ at same $p_{T}$ range. Hence, the precise measurements of ${ }^{3} \mathrm{He}$ and ${ }^{4} \mathrm{He} v_{2}$ in the future can significantly improve the knowledge of the light nuclei production mechanism in heavy-ion collisions.

\section{Conflicts of Interest}

The authors declare that there are no conflicts of interest regarding the publication of this paper. 

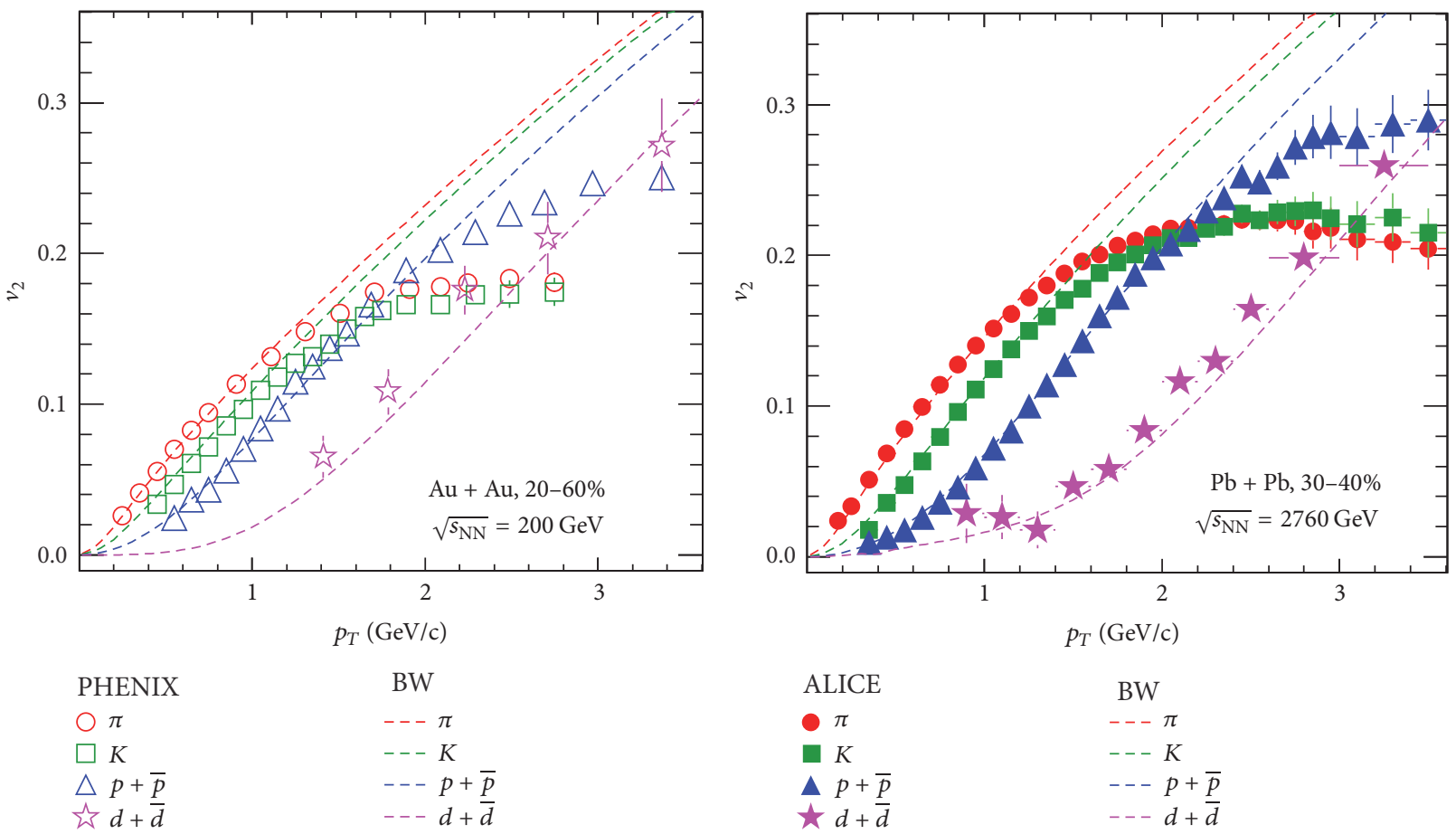

(a)

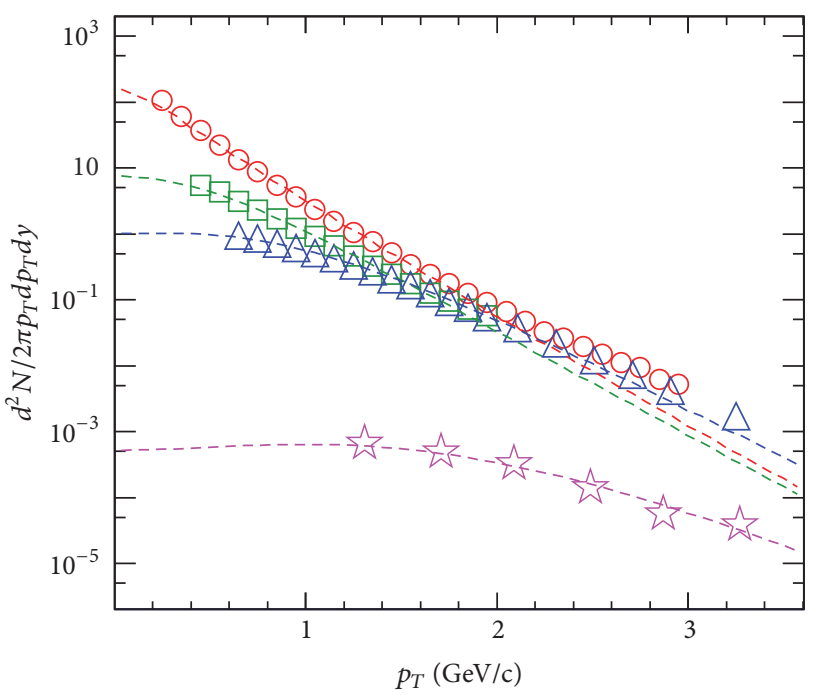
ALICE
$\pi$
BW

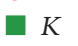
$--K$
$\Delta p+\bar{p}$
$\star d+\bar{d}$
$---p+\bar{p}$
$--d+\bar{d}$

(b)

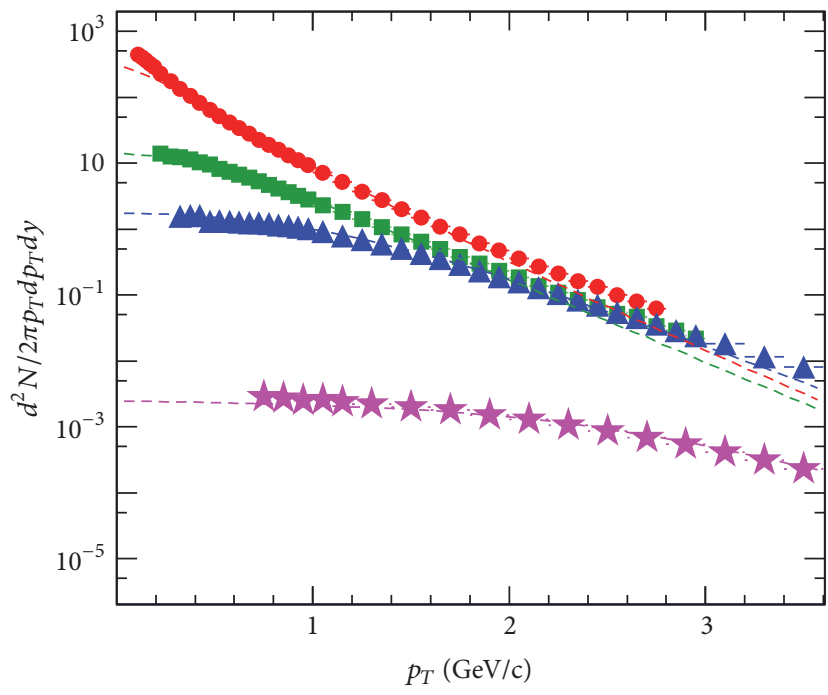

$\begin{array}{ll}\text { PHENIX } & \text { BW } \\ \bigcirc \pi & ---\pi \\ \square K & ---K \\ \triangle p+\bar{p} & --p+\bar{p} \\ \triangle \bar{s} d+\bar{d} & --d+\bar{d}\end{array}$

(c)

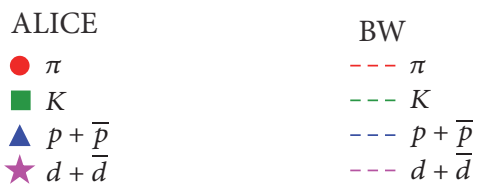

(d)

Figure 9: (a) Blast-wave fit of $\pi, K, p(\bar{p}), d(\bar{d}) v_{2}$, and (c) $p_{T}$ spectra from the PHENIX experiment. The same is shown for the ALICE experiment in panels $(\mathrm{b})$ and $(\mathrm{d}) . p_{T}$ spectra are used from [45, 101]. Marker for PHENIX data corresponds to 20-60\% and marker for ALICE data corresponds to $30-40 \%$ central events. 


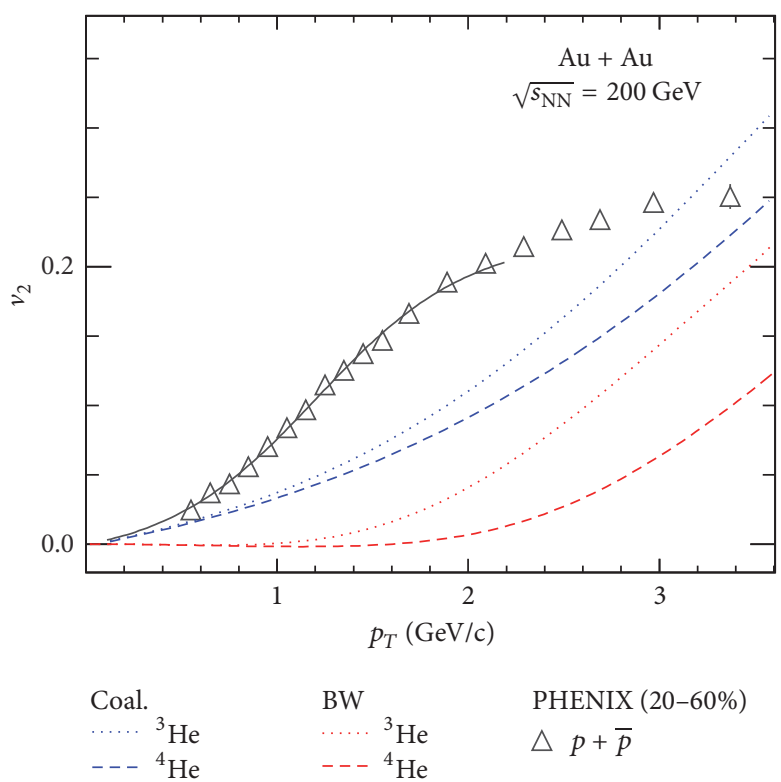

(a)

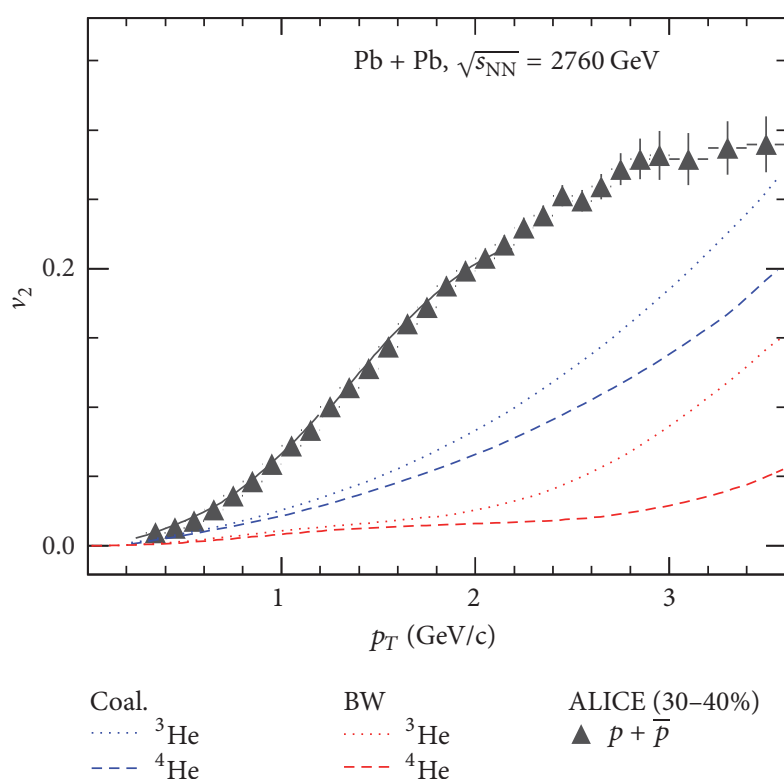

(b)

Figure 10: (a) Coalescence model predictions (blue lines) of ${ }^{3} \mathrm{He}$ and ${ }^{4} \mathrm{He} v_{2}$ for (a) $\sqrt{s_{\mathrm{NN}}}=200 \mathrm{GeV}$ and (b) for $\sqrt{s_{\mathrm{NN}}}=2760 \mathrm{GeV}$. The blast-wave predictions of ${ }^{3} \mathrm{He}$ and ${ }^{4} \mathrm{He} v_{2}$ are also shown in red lines.

\section{Acknowledgments}

The authors thank STAR collaboration, PHENIX collaboration, and ALICE collaboration for providing the light nuclei $v_{2}$ data and the model predictions. This work is supported by DAE-BRNS Project (Grant no. 2010/21/15-BRNS/2026) and Dr. C. Jena is supported by 12 th plan project (PIC no. 12R\&D-NIS-5.11-0300).

\section{References}

[1] M. Stephanov, K. Rajagopal, and E. Shuryak, "Signatures of the tricritical point in QCD," Physical Review Letters, vol. 81, no. 22, pp. 4816-4819, 1998.

[2] M. A. Stephanov, K. Rajagopal, and E. V. Shuryak, "Event-byevent fluctuations in heavy ion collisions and the QCD critical point," Physical Review D, vol. 60, Article ID 114028, 1999.

[3] M. A. Stephanov, "QCD phase diagram: an overview," https:// arxiv.org/abs/hep-lat/0701002.

[4] K. Fukushima and T. Hatsuda, "The phase diagram of dense QCD," Reports on Progress in Physics, vol. 74, no. 1, Article ID 014001, 2010.

[5] I. Arsene, Bearden. I. G., D. Beavis et al., "Quark-gluon plasma and color glass condensate at RHIC? The perspective from the BRAHMS experiment," Nuclear Physics A, vol. 757, no. 1-2, pp. 1-27, 2005.

[6] B. B. Back, M. D. Baker, M. Ballintijn et al., "The PHOBOS perspective on discoveries at RHIC," Nuclear Physics A, vol. 757, pp. 28-101, 2005.

[7] J. Adams, M. M. Aggarwal, Z. Ahammed et al., "Experimental and theoretical challenges in the search for the quark-gluon plasma: The STAR Collaboration's critical assessment of the evidence from RHIC collisions," Nuclear Physics A, vol. 757, no. 1-2, pp. 102-183, 2005.
[8] K. Adcox, S. S. Adler, S. Afanasiev et al., "Formation of dense partonic matter in relativistic nucleus-nucleus collisions at RHIC: experimental evaluation by the PHENIX Collaboration," Nuclear Physics A, vol. 757, no. 1-2, pp. 184-283, 2005.

[9] S. Gupta, X. Luo, B. Mohanty, H. G. Ritter, and N. Xu, "Scale for the phase diagram of quantum chromodynamics," Science, vol. 332, no. 6037, pp. 1525-1528, 2011.

[10] L. Adamczyk, J. K. Adkins, G. Agakishiev et al., "Energy dependence of moments of net-proton multiplicity distributions at RHIC," Physical Review Letters, vol. 112, Article ID 032302, 2014.

[11] N. Itoh, "Hydrostatic equilibrium of hypothetical quark stars," Progress of Theoretical Physics, vol. 44, no. 1, pp. 291-292, 1970.

[12] K. Aamodt, A. Abrahantes Quintana, R. Achenbach et al., "The ALICE experiment at the CERN LHC," Journal of Instrumentation, vol. 3, Article ID S08002, 2008.

[13] B. B. Abelev, J. Adam, D. Adamová et al., "Performance of the ALICE experiment at the CERN LHC," International Journal of Modern Physics A, vol. 29, no. 24, article 120, Article ID 1430044, 2014.

[14] G. Lemaître, "Un Univers homogène de masse constante et de rayon croissant rendant compte de la vitesse radiale des nébuleuses extra-galactiques," Annales de la société Scientifique de Bruxelles, vol. 47, pp. 49-59, 1927.

[15] A.Z. Mekjian, "Explosive nucleosynthesis, equilibrium thermodynamics, and relativistic heavy-ion collisions," Physical Review C, vol. 17, article 1051, 1978.

[16] P. Siemens and J. I. Kapusta, "Evidence for a soft nuclear-matter equation of state," Physical Review Letters, vol. 43, article 1486, 1979.

[17] P. Braun-Munzinger and J. Stachel, "Production of strange clusters and strange matter in nucleus-nucleus collisions at the AGS," Journal of Physics G: Nuclear and Particle Physics, vol. 21, no. 3, article L17, 1995. 
[18] P. Braun-Munzinger and J. Stachel, "Particle ratios, equilibration and the QCD phase boundary," Journal of Physics G: Nuclear and Particle Physics, vol. 28, no. 7, article 1971, 2002.

[19] A. Andronic, P. Braun-Munzinger, J. Stachel, and H. Stocker, "Production of light nuclei, hypernuclei and their antiparticles in relativistic nuclear collisions," Physics Letters B, vol. 697, no. 3, pp. 203-207, 2011.

[20] J. Stachel, A. Andronic, P. Braun-Munzinger, and K. Redlich, "Confronting LHC data with the statistical hadronization model," Journal of Physics: Conference Series, vol. 509, no. 1, Article ID 01201, 2014.

[21] S. Chatterjee and B. Mohanty, "Production of light nuclei in heavy-ion collisions within a multiple-freezeout scenario," Physical Review C, vol. 90, Article ID 034908, 2014.

[22] S. T. Butler and C. A. Pearson, "Deuterons from high-energy proton bombardment of matter," Physical Review Letters, vol. 129, no. 836, 1963.

[23] A. Schwarzschild and A. Zupancic, "Production of Tritons, Deuterons, Nucleons, and Mesons by $30-\mathrm{GeV}$ Protons on A1, Be, and Fe targets," Physical Review Letters, vol. 129, no. 2, pp. 854-862, 1963.

[24] H. H. Gutbrod, A. Sandoval, P. J. Johansen et al., "Final-state interactions in the production of hydrogen and helium isotopes by relativistic heavy ions on uranium," Physical Review Letters, vol. 37, 667 pages, 1976.

[25] H. Sato and K. Yazaki, "On the coalescence model for high energy nuclear reactions," Physics Letters B, vol. 98, no. 3, pp. 153-157, 1981.

[26] E. A. Remler, "Composite particle cross sections from the density operator," Annals of Physics, vol. 136, no. 2, pp. 293-316, 1981.

[27] M. Gyulassy, K. Frankel, and E. A. Remler, "Deuteron formation in nuclear collisions," Nuclear Physics A, vol. 402, no. 3, pp. 596611, 1983.

[28] L. Csernai and J. I. Kapusta, "Entropy and cluster production in nuclear collisions," Physics Reports, vol. 131, no. 4, pp. 223-318, 1986.

[29] P. Danielewicz and G. F. Bertsch, "Production of deuterons and pions in a transport model of energetic heavy-ion reactions," Nuclear Physics A, vol. 533, no. 4, pp. 712-748, 1991.

[30] C. B. Dover, U. Heinz, E. Schnedermann, and J. Zimnyi, "Covariant coalescence model for relativistically expanding systems," Physical Review C, vol. 44, no. 4, pp. 1636-1654, 1991.

[31] W. J. Llope, S. E. Pratt, N. Frazier et al., "The fragment coalescence model," Physical Review C, vol. 52, article 2004, 1995.

[32] J. L. Nagle, B. S. Kumar, D. Kusnezov, H. Sorge, and R. Mattiello, "Coalescence of deuterons in relativistic heavy ion collisions," Physical Review C, vol. 53, no. 1, pp. 367-376, 1996.

[33] R. Scheibl and U. Heinz, "Coalescence and flow in ultrarelativistic heavy ion collisions," Physical Review C, vol. 59, article 1585, 1999.

[34] S. Zhang, J. Chen, H. Crawford, D. Keane, Y. Ma, and Z. B. $\mathrm{Xu}$, "Searching for onset of deconfinement via hypernuclei and baryon-strangeness correlations," Physics Letters B, vol. 684, no. 4-5, pp. 224-227, 2010.

[35] J. Steinheimer, K. Gudima, A. Botvina, I. Mishustin, M. Bleicher, and H. Stöcker, "Hypernuclei, dibaryon and antinuclei production in high energy heavy ion collisions: thermal production vs. coalescence," Physics Letters B, vol. 714, no. 1, pp. 85-91, 2012.
[36] S. Mrówczyński, "Production of light nuclei in the thermal and coalescence models," Acta Physica Polonica B, vol. 48, no. 4, p. 707, 2017.

[37] V. I. Voloshin, "Anisotropic flow," Nuclear Physics A, vol. 715, pp. 379c-388c, 2003.

[38] D. Molnar and S. A. Voloshin, "Elliptic flow at large transverse momenta from quark coalescence," Physical Review Letters, vol. 91, Article ID 092301, 2003.

[39] R. C. Hwa and C. B. Yang, "Scaling distributions of quarks, mesons, and proton for all PT energy, and centrality," Physical Review C, vol. 67, no. 6, Article ID 064902, 2003.

[40] R. J. Fries, B. Muller, C. Nonaka, and S. A. Bass, "Hadronization in heavy-ion collisions: recombination and fragmentation of partons," Physical Review Letters, vol. 90, Article ID 202303, 2003.

[41] L. Adamczyk, J. K. Adkins, G. Agakishiev et al., "Elliptic flow of identified hadrons in $\mathrm{Au}+\mathrm{Au}$ collisions at $\sqrt{s N N}=7.7-62.4$ GeV," Physical Review C, vol. 88, Article ID 014902, 2013.

[42] L. Adamczyk, J. K. Adkins, G. Agakishiev et al., "Observation of an energy-dependent difference in elliptic flow between particles and anti-particles in relativistic heavy ion collisions," Physical Review Letters, vol. 110, Article ID 142301, 2013.

[43] L. Adamczyk, J. K. Adkins, G. Agakishiev et al., "Centrality and transverse momentum dependence of elliptic flow of multistrange hadrons and $\varnothing$ Meson in $\mathrm{Au}+\mathrm{Au}$ Collisions at $\sqrt{s \mathrm{NN}}=200 \mathrm{GeV}$," Physical Review Letters, vol. 116, no. 6, Article ID 062301, 2016.

[44] S. S. Adler, Z. Ahammed, C. Allgower et al., "Identified particle elliptic flow in Au+Au collisions at $\sqrt{s \mathrm{NN}}=200 \mathrm{GeV}$," Physical Review Letters, vol. 91, Article ID 182301, 2003.

[45] S. S. Adler, S. Afanasiev, C. Aidala et al., "Identified charged particle spectra and yields in $\mathrm{Au}+\mathrm{Au}$ collisions at $\sqrt{s \mathrm{NN}}=200$ GeV," Physical Review C, vol. 69, Article ID 034909, 2004.

[46] S. Nagamiya, M.-C. Lemaire, E. Moeller et al., "Production of pions and light fragments at large angles in high-energy nuclear collisions," Physical Review C, vol. 24, article 971, 1981.

[47] R. L. Auble, J. B. Ball, F. E. Bertrand et al., "Light ion emission from reactions induced by $0.8-2.4 \mathrm{GeV}^{16} \mathrm{O}$ projectiles," Physical Review C, vol. 28, article 1552, 1983.

[48] S. Wang, S. Albergo, F. Bieser et al., "Light fragment production and power law behavior in Au + Au collisions," Physical Review Letters, vol. 74, article 2646, 1995.

[49] M. A. Lisa, S. Albergo, F. Bieser et al., "Radial flow in $\mathrm{Au}+\mathrm{Au}$ collisions at $E=(0.25-1.15) A \mathrm{GeV}$," Physical Review Letters, vol. 75, article 2662, 1995.

[50] M. Aoki, J. Beatty, D. Beavis et al., "Measurements at $0^{\circ}$ of negatively charged particles and antinuclei produced in collisions of 14.6A GeV/c Si on Al, Cu, and Au targets," Physical Review Letters, vol. 69, article 2345, 1992.

[51] T. A. Armstrong, K. N. Barish, S. Batsouli et al., "Antideuteron yield at the AGS and coalescence implications," Physical Review Letters, vol. 85, article 2685, 2000.

[52] J. Barrette, R. Bellwied, S. Bennett et al., "Light fragment yields from central $\mathrm{Au}+\mathrm{Au}$ collisions at 11.5A GeV/c," Physical Review C, vol. 61, Article ID 044906, 2000.

[53] S. Albergo, R. Bellwied, M. Bennett et al., "Light nuclei production in heavy-ion collisions at relativistic energies," Physical Review C, vol. 65, Article ID 034907, 2002.

[54] S. Kabana, G. Ambrosini, R. Arsenescu et al., "New results from NA52 on particle production in $\mathrm{Pb}+\mathrm{Pb}$ collisions at $158 \mathrm{GeV}$ per nucleon," Nuclear Physics A, vol. 638, no. 1-2, pp. 411c-414c, 1998. 
[55] T. Anticic, R. Snellings, and S. Voloshin, "Flow analysis with cumulants: direct calculations," Physical Review C, vol. 85, Article ID 044913, 2012.

[56] G. L. Melkumov, "Recent results on (anti)nucleus and (anti)hyperon production in nucleus-nucleus collisions at CERN SPS energies," PoS(CPOD07), 024, 2007.

[57] V. I. Kolesnikov, "Anti-nuclei and nuclei production in $\mathrm{Pb}+\mathrm{Pb}$ collisions at CERN SPS energies," Journal of Physics: Conference Series, vol. 110, no. 3, Article ID 032010, 2008.

[58] I. G. Bearden, H. Bøggild, J. G. Boissevain, and A. Ljubicic, "Antideuteron production in $158 \mathrm{~A} \mathrm{GeV} / \mathrm{c} \mathrm{Pb}+\mathrm{Pb}$ collisions," Physical Review Letters, vol. 85, article 2681, 2000.

[59] A. Polleri, J. P. Bondorf, I. N. Mishustin et al., "Effects of collective expansion on light cluster spectra in relativistic heavy ion collisions," Physics Letters B, vol. 419, pp. 19-24, 1998.

[60] S. Voloshin and Y. Zhang, "Flow study in relativistic nuclear collisions by Fourier expansion of azimuthal particle distributions," Zeitschrift für Physik C Particles and Fields, vol. 70, no. 4, pp. 665-671, 1996.

[61] A. M. Poskanzer and S. A. Voloshin, "Methods for analyzing anisotropic flow in relativistic nuclear collisions," Physical Review C, vol. 58, article 1671, 1998.

[62] P. Danielewicz, "Effects of compression and collective expansion on particle emission from central heavy-ion reactions," Physical Review C, vol. 51, article 716, 1995.

[63] L. Adamczyk, J. K. Adkins, G. Agakishiev et al., "Measurement of elliptic flow of light nuclei at $\sqrt{s_{\mathrm{NN}}}=200,62.4,39,27$, 19.6, 11.5, and $7.7 \mathrm{GeV}$ at the BNL relativistic heavy ion collider," Physical Review C, vol. 94, Article ID 034908, 2016.

[64] J. Barrette et al., "Proton and pion production relative to the reaction plane in $\mathrm{Au}+\mathrm{Au}$ collisions at $11 \mathrm{AGeV} / c$," Physical Review C, vol. 56, article 3254, 1997.

[65] J. Barrette, R. Bellwied, S. Bennett et al., "Energy and charged particle flow in $10.8 \mathrm{~A} \mathrm{GeV/c} \mathrm{Au+Au} \mathrm{collisions,"} \mathrm{Physical} \mathrm{Review}$ C, vol. 55, article 1420, 1997.

[66] S. Afanasiev et al., "Elliptic Flow for $\phi$ Mesons and (Anti)deuterons in $\mathrm{Au}+\mathrm{Au}$ Collisions at $\sqrt{s N N}=200$ GeV," Physical Review Letters, vol. 99, Article ID 052301, 2007.

[67] S. Acharya, D. Adamová, J. Adolfsson et al., "Measurement of deuteron spectra and elliptic flow in $\mathrm{Pb}-\mathrm{Pb}$ collisions at $\sqrt{s_{\mathrm{NN}}}=$ 2.76 TeV at the LHC," https://arxiv.org/abs/1707.07304.

[68] M. Anderson, J. Berkovitz, W. Betts et al., "The STAR time projection chamber: a unique tool for studying high multiplicity events at RHIC," Nuclear Instruments and Methods in Physics Research Section A, vol. 499, pp. 659-678, 2003.

[69] J. Adams, M. M. Aggarwal, Z. Ahammed et al., "Identified hadron spectra at large transverse momentum in $p+p$ and $d$ + Au collisions at $\sqrt{s N N}=200 \mathrm{GeV}$,' Physics Letters B, vol. 637, pp. 161-169, 2006.

[70] J. Alme, Y. Andres, H. Appelshäuser et al., "The ALICE TPC, a large 3-dimensional tracking device with fast readout for ultrahigh multiplicity events," Nuclear Instruments and Methods in Physics Research Section A, vol. 622, pp. 316-367, 2010.

[71] J. Adam, D. Adamová, M. M. Aggarwal et al., "Production of light nuclei and anti-nuclei in $\mathrm{pp}$ and $\mathrm{Pb}-\mathrm{Pb}$ collisions at $\mathrm{LHC}$ energies," Physical Review C, vol. 93, Article ID 024917, 2016.

[72] W. J. Llope and STAR TOF Group, "The large-area time-of-flight upgrade for STAR," Nuclear Instruments and Methods in Physics Research Section B: Beam Interactions with Materials and Atoms, vol. 241, p. 306, 2005.
[73] W. J. Llope, "Multigap RPCs in the STAR experiment at RHIC," Nuclear Instruments and Methods in Physics Research A, vol. 661, pp. S110-S113, 2012.

[74] A. Akindinov, A. Alici, A. Agostinelli et al., "Performance of the ALICE time-of-flight detector at the LHC," The European Physical Journal Plus, vol. 128, no. 44, 2013.

[75] B. Abelev, J. Adam, D. Adamová et al., "Elliptic flow of identified hadrons in $\mathrm{Pb}-\mathrm{Pb}$ collisions at $\sqrt{s N N}=2.76 \mathrm{TeV}$," Journal of High Energy Physics, vol. 2015, article 190, 2015.

[76] P. Huovinen, P. F. Kolb, U. W. Heinz, P. V. Ruuskanen, and S. A. Voloshin, "Radial and elliptic flow at RHIC: further predictions," Physics Letters B, vol. 503, no. 1-2, pp. 58-64, 2001.

[77] H. Li, L. He, Z.-W. Lin, D. Molnar, F. Wang, and W. Xie, "Origin of the mass splitting of elliptic anisotropy in a multiphase transport model," Physical Review C, vol. 93, Article ID 051901, 2016.

[78] H. Li, L. He, Z.-W. Lin, D. Molnar, F. Wang, and W. Xie, “Origin of the mass splitting of azimuthal anisotropies in a multi-phase transport model," https://arxiv.org/abs/1604.07387.

[79] H. Xu, Z. Li, and H. Song, "High-order flow harmonics of identified hadrons in $2.76 \mathrm{~A} \mathrm{TeV} \mathrm{Pb}+\mathrm{Pb}$ collisions," Physical Review C, vol. 93, Article ID 064905, 2016.

[80] S. A. Voloshin, A. M. Poskanzer, and R. Snellings, Collective Phenomena in Non-Central Nuclear Collisions, vol. 23 of LandoltBörnstein Series, Springer, Berlin, Germany, 2010.

[81] Y. Burnier, D. E. Kharzeev, J. Liao, and H. U. Yee, "Chiral magnetic wave at finite baryon density and the electric quadrupole moment of the quark-gluon plasma," Physical Review Letters, vol. 107, Article ID 052303, 2011.

[82] J. C. Dunlop, M. A. Lisa, and P. Sorensen, "Constituent quark scaling violation due to baryon number transport," Physical Review C, vol. 84, Article ID 044914, 2011.

[83] V. Greco, M. Mitrovski, and G. Torrieri, "Elliptic flow in heavy ion collisions at varying energies: partonic versus hadronic dynamics," Physical Review C, vol. 86, Article ID 044905, 2012.

[84] J. Steinheimer, V. Koch, and M. Bleicher, "Hydrodynamics at large baryon densities: understanding proton versus antiproton $v_{2}$ and other puzzles," Physical Review C, vol. 86, Article ID 044903, 2012.

[85] J. Xu, L. W. Chen, C. M. Ko, and Z. W. Lin, "Effects of hadronic potentials on elliptic flows in relativistic heavy ion collisions," Physical Review C, vol. 85, Article ID 041901, 2012.

[86] J. Xu, T. Song, C. M. Ko, and F. Li, "Elliptic flow splitting as a probe of the QCD phase structure at finite baryon chemical potential," Physical Review Letters, vol. 112, Article ID 012301, 2014.

[87] T. Song, S. Plumari, V. Greco, C. M. Ko, and F. Li, "Partonic mean-field effects on matter and antimatter elliptic flows," https://arxiv.org/abs/1211.5511.

[88] J. Xu, C. M. Ko, F. Li, T. Song, and H. Liu, "Mean-field potential effects on particle and antiparticle elliptic flows in the beamenergy scan program at RHIC," Nuclear Physics Review, vol. 32, article 146, 2015.

[89] L. Adamczyk, J. K. Adkins, G. Agakishiev et al., "Centrality dependence of identified particle elliptic flow in relativistic heavy ion collisions at $\sqrt{s N N}=7.7-62.4 \mathrm{GeV}$," Physical Review C, vol. 93, Article ID 014907, 2016.

[90] B. Abelev, J. Adam, D. Adamová et al., "Elliptic flow of identified hadrons in $\mathrm{Pb}-\mathrm{Pb}$ collisions at $\sqrt{s N N}=2.76 \mathrm{TeV}$," Journal of High Energy Physics, vol. 2015, article 190, 190 pages, 2015. 
[91] T. Z. Yan, Y. G. Ma, X. Z. Cai et al., "Scaling of anisotropic flow and momentum-space densities for light particles in intermediate energy heavy ion collisions," Physics Letters B, vol. 638, no. 1, pp. 50-54, 2006.

[92] Y. Oh and C. M. Ko, "Elliptic flow of deuterons in relativistic heavy-ion collisions," Physical Review C - Nuclear Physics, vol. 76, no. 5, Article ID 054910, 2007.

[93] X. Sun, H. Masui, A. M. Poskanzer, and A. Schmah, "Blast wave fits to elliptic flow data at $\sqrt{s_{\mathrm{NN}}}=7.7-2760 \mathrm{GeV}$," Physical Review C, vol. 91, Article ID 024903, 2015.

[94] Z. Lin, C. M. Ko, B. A. Li, B. Zhang, and S. Pal, "Multiphase transport model for relativistic heavy ion collisions," Physical Review C, vol. 72, Article ID 064901, 2005.

[95] M. R. Haque, Nuclei production and azimuthal anisotropy of charged particles in heavy-ion collisions at RHIC [Ph.D Thesis], NISER India, https://drupal.star.bnl.gov/STAR/theses/phd-67.

[96] L. Zhu, C. M. Ko, and X. Yin, "Light (anti-)nuclei production and flow in relativistic heavy-ion collisions," Physical Review C, vol. 92, no. 6, Article ID 064911, 2015.

[97] X. Dong, S. Esumi, P. Sorensen, N. Xu, and Z. Xu, "Resonance decay effects on anisotropy parameters," Physics Letters B, vol. 597, no. 3-4, pp. 328-332, 2004.

[98] H. Agakishiev, A. V. Alakhverdyants, G. S. Averichev et al., "Observation of the antimatter helium-4 nucleus," Nature, vol. 473, pp. 353-356, 2011.

[99] B. I. Abelev, M. M. Aggarwal, and Z. Ahammed, "Observation of an antimatter hypernucleus," Science, vol. 328, no. 5974, pp. 58-62, 2010.

[100] J. Adam, D. Adamová, M. M. Aggarwal et al., “ ${ }_{A}^{3} H$ and production in $\mathrm{Pb}-\mathrm{Pb}$ collisions at $\sqrt{s N N}=2.76 \mathrm{TeV}$," Physics Letters B, vol. 754, pp. 360-372, 2016.

[101] B. Abelev, J. Adam, D. Adamová et al., "Production of charged pions, kaons and protons at large transverse momenta in $p p$ and Pb-Pb collisions at $\sqrt{s N N}=2.76 \mathrm{TeV}$," Physics Letters B, vol. 736, pp. 196-207, 2014. 

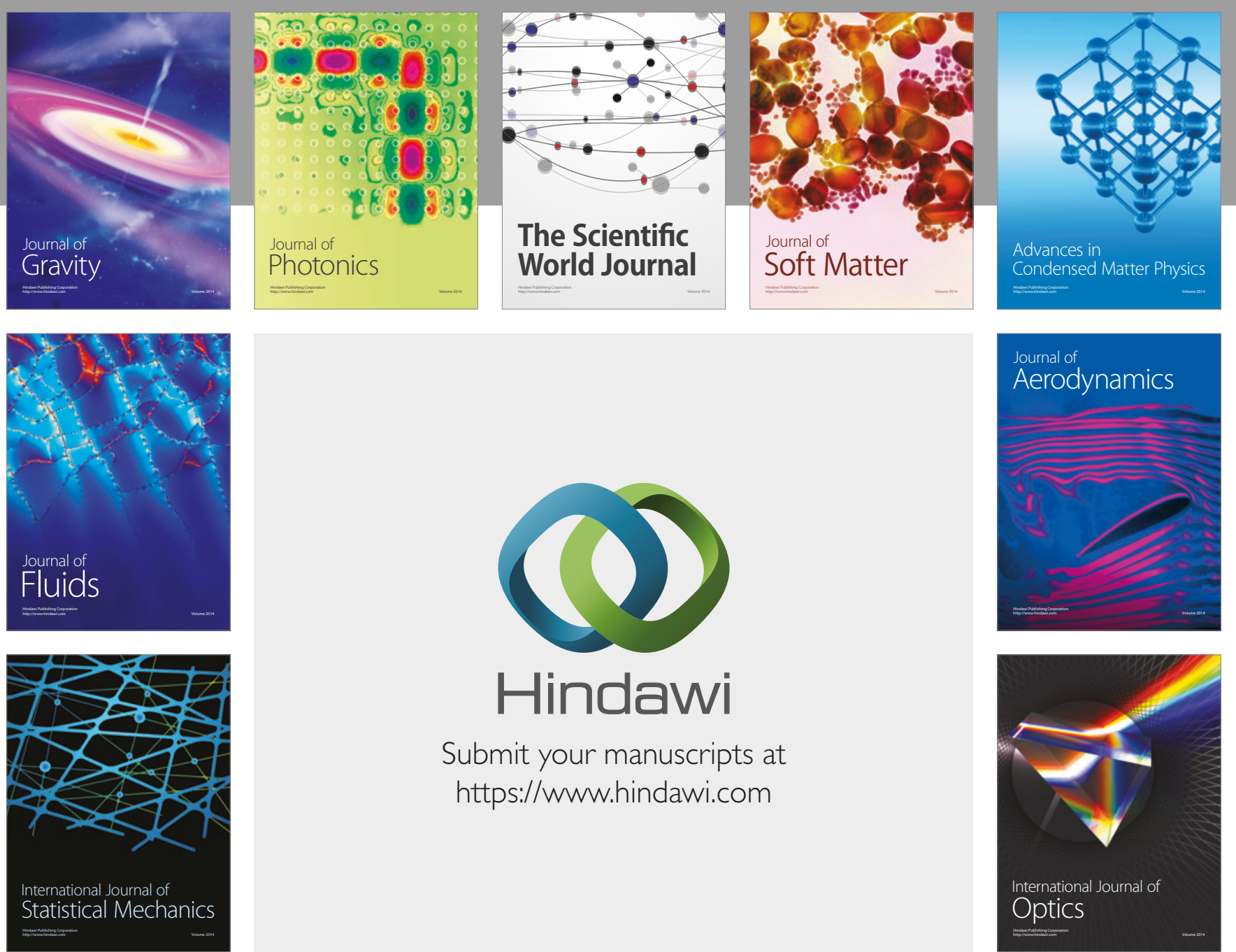

Submit your manuscripts at

https://www.hindawi.com
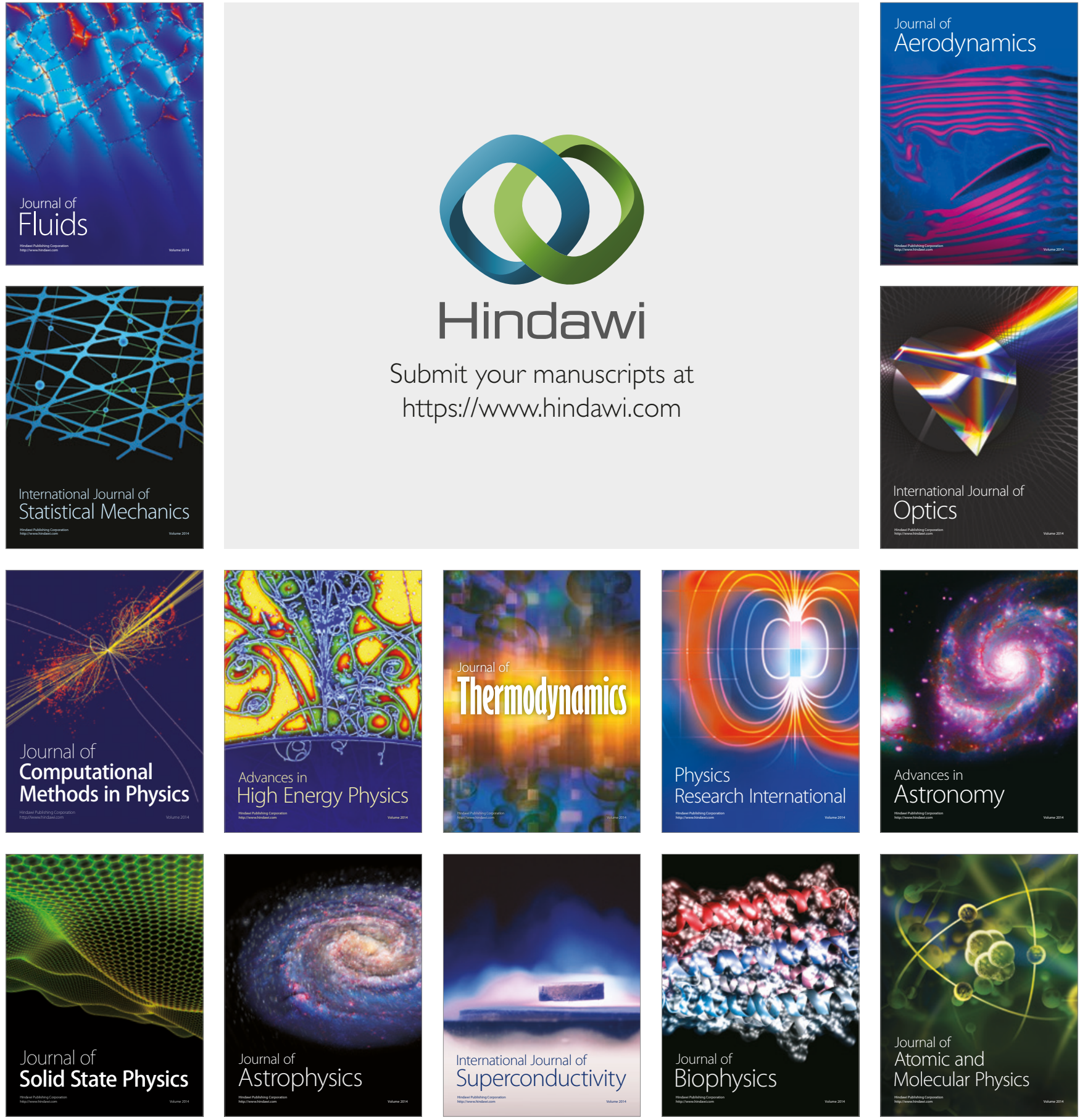